\title{
Quadrupole moments, edge polarizations, and corner charges in the Wannier representation
}

\author{
Shang Ren, ${ }^{1}$ Ivo Souza, ${ }^{2,3}$ and David Vanderbilt ${ }^{1}$ \\ ${ }^{1}$ Department of Physics \& Astronomy, Rutgers University, Piscataway, New Jersey 08854, USA \\ ${ }^{2}$ Centro de Física de Materiales, Universidad del País Vasco, 20018 San Sebastián, Spain \\ ${ }^{3}$ Ikerbasque Foundation, 48013 Bilbao, Spain
}

\begin{abstract}
The modern theory of polarization allows for the determination of the macroscopic end charge of a truncated one-dimensional insulator, modulo the charge quantum $e$, from a knowledge of bulk properties alone. A more subtle problem is the determination of the corner charge of a two-dimensional insulator, modulo $e$, from a knowledge of bulk and edge properties alone. While previous works have tended to focus on the quantization of corner charge in the presence of symmetries, here we focus on the case that the only bulk symmetry is inversion, so that the corner charge can take arbitrary values. We develop a Wannier-based formalism that allows the corner charge to be predicted, modulo $e$, only from calculations on ribbon geometries of two different orientations. We elucidate the dependence of the interior quadrupole and edge dipole contributions upon the gauge used to construct the Wannier functions, finding that while these are individually gauge-dependent, their sum is gauge-independent. From this we conclude that the edge polarization is not by itself a physical observable, and that any Wannier-based method for computing the corner charge requires the use of a common gauge throughout the calculation. We satisfy this constraint using two Wannier construction procedures, one based on projection and another based on a gauge-consistent nested Wannier construction. We validate our theory by demonstrating the correct prediction of corner charge for several tight-binding models. We comment on the relations between our approach and previous ones that have appeared in the literature.
\end{abstract}

\section{INTRODUCTION}

From elementary electrostatics it is well known that the electric polarization in an insulator, corresponding to the dipole density, gives rise to bound charges at the surface. However, the definition of bulk dipole density is not obvious in the context of a quantum treatment of the electron system, since the electron charge cloud is not naturally decomposable into localized entities. This problem was solved by the modern theory of polarization, which can be formulated in the single-particle context either in terms of Berry phases of the Bloch functions, or in terms of dipole moments of Wannier functions (WFs) [1$3]$.

Adopting the latter point of view, the polarization is defined in terms of the dipole moment of the unit cell, taken to consist of point ionic charges and the continuous but exponentially localized charge clouds of the WFs attached to that cell. Crucially, although gauge transformations of the Bloch functions result in changes of both the shapes and charge centers of the WFs, the vector sum of the Wannier centers in one unit cell is gauge-invariant up to a lattice vector. As a result, the polarization is well defined modulo a quantum $e \mathbf{R} / V_{\text {cell }}$, where $e$ is the quantum of charge, $\mathbf{R}$ is a real-space lattice vector, and $V_{\text {cell }}$ is the unit cell volume.

Recently, several groups have explored generalizations of this theory to the quadrupole and higher moments of the charge distributions in insulating crystalline solids. Benalcazar, Bernevig and Hughes $[4,5]$ introduced the concept of "topological quadrupole insulators," in which the corner charge is quantized by symmetries, as examples of "higher-order topological insulators" [6]. This work attracted considerable attention. Several authors adopted a Wannier (or hybrid Wannier) representation as a means to define the topological indices in such higher-order topological insulators [7-11]. Attempts were put forward to derive a formula for the corner charge, either when it is quantized by symmetries $[8,12-14]$, or in the more general case where it takes a nonquantized value [15]. It was shown that even common ionic compounds such as $\mathrm{NaCl}$ may display a fractional corner charge [16]. Other works $[17,18]$ attempted to extend a quadrupole-moment expression to the many-body case by making use of Resta's position operator formalism [19], but these approaches have proven to be controversial $[13,20]$.

Most of these previous works have mainly been concerned with systems whose symmetry quantizes the corner charges. In the absence of symmetry, however, it is unclear whether a robust definition of a bulk quadrupole density, analogous to that of the electric polarization for the dipole density, is possible, even at the singleparticle level $[13,17,18,20]$. The essential problem is that unlike the total dipole of the Wannier charge distribution associated with a unit cell, the corresponding quadrupole is not gauge-invariant. In fact, the trace of the Wannier quadrupole is essentially the spread functional that is minimized when arriving at maximally localized WFs [21, 22]; the very fact that it can be minimized is a reflection of its gauge dependence. It is not surprising, then, that the off-diagonal elements of the quadrupole tensor are also gauge-dependent, i.e., they vary according to the exact locations and shapes of the WFs. For this reason, the theory of quadrupoles and higher multipoles is fundamentally different from the the- 
ory of dipoles that underlies the modern theory of polarization.

Just as a bulk dipole density results in a bound surface charge, so a bulk quadrupole density is expected to result in bound surface polarizations and edge charges in $3 \mathrm{D}$, or edge polarizations and corner charges in $2 \mathrm{D}[4,5,15,23]$, where it is understood that we refer to the polarization tangential to the surface or edge. Intuitively, a quadrupole density $\mathcal{Q}_{x y}$ in a 2 D sample results in bound 1D dipole densities $\mathcal{P}_{x}=\mathcal{Q}_{x y}$ at the $+\hat{\mathbf{y}}$-normal edge and $\mathcal{P}_{y}=\mathcal{Q}_{x y}$ at the $+\hat{\mathbf{x}}$-normal edge. It also results in an overall bound charge $Q_{\mathrm{c}}=\mathcal{Q}_{x y}$ at the corner where these edges meet, but this $Q_{\mathrm{c}}$ is not simply the sum of the contributions expected from the edge polarizations. Thus, such definitions become quite subtle, even for simple classical charge distributions $[4,5,15]$.

In fact, there are serious reasons to question whether the edge polarization is a physical observable at all. We give two arguments that it is not. To do so, we focus on a large rectangular flake cut from an insulating 2D crystal, and frame the discussion in terms of spinless electrons.

First, recall that in the case of dipole densities, there is a robust bulk-boundary correspondence in that the macroscopic edge charge density is exactly given by the bulk polarization projected onto the edge unit normal, modulo a quantum of one electron per edge unit cell [24]. This means that no adiabatic periodicity-preserving perturbation at the edge, such as a displacement of a sublattice of edge atoms, can have any effect whatsoever on the edge charge density. It is natural, then, to regard the macroscopic edge charge density as a manifestation of a bulk property. The edge dipole density, on the other hand, is obviously modified by such edge-atom displacements, suggesting that it is not a manifestation of a bulk property in the same sense.

Second, insofar as a $1 \mathrm{D}$ polarization $\mathcal{P}$ is well defined, we would expect its time derivative $d \mathcal{P} / d t$ to correspond to a physically observable edge current. However, this is problematic in the case of edge polarizations and currents. For example, if the insulating flake in question has been cut from a bulk that has some nonzero orbital magnetization $M_{\text {orb }}$ (as a consequence of broken timereversal symmetry), then there will be a persistent counterclockwise current $I=M_{\text {orb }}$ on each edge, forcing the nonsensical conclusion that $\mathcal{P}$ increases linearly in time. In fact, even if the bulk material itself is time-reversal invariant, so that its intrinsic orbital magnetization vanishes, Trifunovic, Ono, and Watanabe [25] have shown that when such a system is carried adiabatically around a parametric loop, this results in a net circulation of current around the perimeter of the sample. This would imply that the edge polarization can be changed by an arbitrary amount by such an adiabatic cycle. These arguments suggest that any attempt to define the change in edge polarization in terms of an integrated current, as is done for the bulk polarization, is bound to run into grave difficulties.

The arguments given above imply that there are se- rious difficulties associated with attempts to define the bulk quadrupole density and edge dipole density in a 2D system. By contrast, the macroscopic corner charge is unambiguously a physical observable. Thus, given details of the geometric structure and the electronic Hamiltonian of the 1D-periodic edges as well as of the 2D-periodic bulk, a robust theory should be capable of correctly predicting the macroscopic corner charges modulo $e$.

In this work, we show how to construct such a theory for the case of centrosymmetric $2 \mathrm{D}$ insulators, based on a Wannier representation of the electronic system at the single-particle level. In our formulation, we first identify a bulk unit cell, or "tile," composed of a set of ionic positive point charges and the charge distributions associated with a set of bulk WFs. The quadrupole density $\mathcal{Q}_{x y}$ associated with this unit cell is gauge-dependent, i.e., dependent on the exact locations and shapes of WFs in the unit cell. We also construct "edge tiles" consisting of ions and WFs in a "skin" region close to the edge, and associate surface polarizations $\mathcal{P}$ to these edges. In our formulation the edge $\mathcal{P}$ 's are defined independently of the bulk $\mathcal{Q}_{x y}$, as they must be since they depend upon the detailed form of the Hamiltonian at the edge. While the $\mathcal{P}$ 's are independent of a gauge change localized at the edge, they are, like $\mathcal{Q}_{x y}$, dependent on the choice of bulk WF gauge. Nevertheless, we find that all gauge dependence cancels out when the various contributions are summed, thus allowing for a robust prediction of the corner charge.

Specifically, we work in the context of tight-binding models of centrosymmetric 2D insulators whose bulk and edge electronic structures are gapped. We solve for the ground-state electronic structure in four configurations, namely the infinite bulk with 2D periodic boundary conditions, 1D-periodic ribbons of finite width in the $x$ direction, the same but finite in the $y$ direction, and rectangular flakes with fully open boundary conditions. We develop two formalisms for computing the macroscopic corner charge $(\bmod e)$ from the bulk and ribbon calculations alone, and demonstrate their success by direct calculation on the rectangular flake.

In the course of preparing this manuscript, we became aware of related work of Trifunovic [15], in which similar questions are addressed from a somewhat different point of view. While that work considers more general unit cell shapes and corner geometries than we do, the implementation was only presented for the case of single-occupiedband models and for the isolated molecular limit of the Benalcazar-Bernevig-Hughes model $[4,5]$. We occasionally comment on similarities and differences below.

This paper is organized as follows. In Sec. II, we introduce an expression for the macroscopic corner charge in terms of contributions from bulk, edge, and corner charge densities based on a tiling approach. We explain how quadrupole, dipole, and monopole contributions from bulk, edge, and corner tiles, respectively, add up to give the observable macroscopic corner charge. In this formulation, the electronic charge density associated 
with each tile is that of the WFs attached to it, raising questions about the dependence of the bulk and edge contributions on the gauge used to construct these WFs. This issue is addressed in Sec. III, where we show that the sum of bulk and edge contributions is indeed gaugeinvariant, even though the individual contributions are not. In Sec. IV, we provide additional details about our methodology. Specifically, in Sec. IV A we discuss how we calculate the macroscopic corner charge directly from a finite flake. Then in Sec. IV B we present several approaches to the construction of Wannier functions for ribbon models, including a projection approach (Sec. IV B 1) and approaches based on maximal localization applied first transverse (Sec. IV B 2) or parallel (Sec. IV B 3) to the extended ribbon direction. We then demonstrate in Sec. V the limitations of a naive hybrid Wannier implementation, and show that these are overcome using the gauge-consistent projection method, for three centrosymmetric tight-binding models at half filling. Specifically, we consider a two-band model [23], a related four-band model, and the four-band model proposed in Refs. [4] and [5] to discuss quantization of the corner charges. In Sec. VI, we present a nested maximally-localized Wannier construction that also generates a consistent gauge, and working in the context of the four-band model, show that this also provides a correct prediction of the corner charge. We discuss some possible generalizations of our approach and its relation to the theory of orbital magnetization in Sec. VII, and summarize in Sec. VIII.

\section{PRELIMINARIES}

\section{A. General considerations from tiling}

We consider a centrosymmetric $2 \mathrm{D}$ crystalline material having a rectangular unit cell with lattice vectors $\mathbf{a}=a \hat{\mathbf{x}}$ and $\mathbf{b}=b \hat{\mathbf{y}}$. A finite sample, or "flake," has been cut from this material, and its charge density is assumed to be written as the sum of $N_{x} \times N_{y}$ contributions from the individual unit cells. In the deep interior all these cells are identical, but those near the edges and corners are modified by the presence of the boundaries.

We identify a "skin region" on each edge, consisting of $M_{y}$ cells at top and bottom and $M_{x}$ cells at left and right, where $M_{x}$ and $M_{y}$ are chosen large enough that the deeper tiles are bulk-like to some desired accuracy. This is illustrated in Fig. 1 , where $M_{x}=M_{y}=2$. We decompose the charge density of the flake as a whole into contributions from the interior, the four skin regions, and the four left-over corner regions. That is, we write

$$
\rho_{\text {flake }}(\mathbf{r})=\rho^{\mathrm{I}}(\mathbf{r})+\rho^{\mathrm{S}}(\mathbf{r})+\rho^{\mathrm{C}}(\mathbf{r})
$$

where the superscripts denote "interior," "skin," and "corner" contributions (black, red, and blue regions in Fig. 1), respectively.

The first term in Eq. (1) is the superposition of the

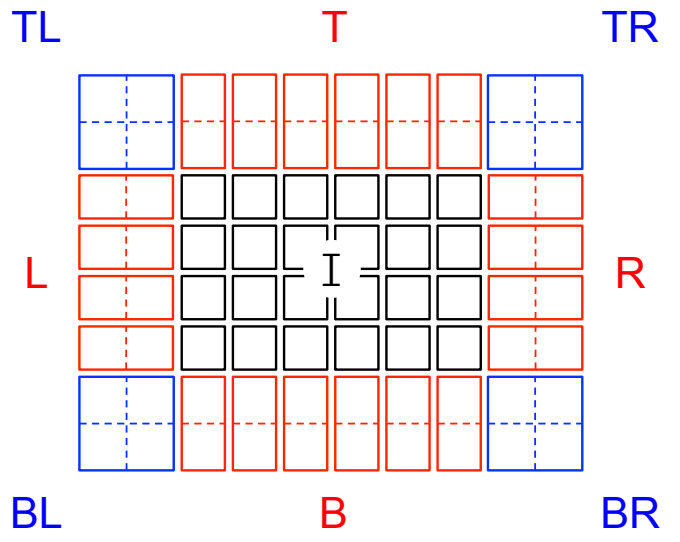

FIG. 1. Sketch of tiling scheme for a rectangular sample cut from a 2D crystal. Small square tiles (black) correspond to single interior ('I') unit cells. Rectangular edge tiles (red) and larger corner tiles (blue), which may extend to a depth of two or more cells, define the skin region. Edges are labeled as ' $\mathrm{T}$ ' (top), 'R' (right), 'B' (bottom), and 'L' (left), and corners are labeled by combinations such as 'TR' (top right).

identical interior tile charge densities, i.e.,

$$
\rho^{\mathrm{I}}(\mathbf{r})=\sum_{\ell_{x} \in I_{x}} \sum_{\ell_{y} \in I_{y}} \rho_{\text {tile }}^{\mathrm{I}}\left(\mathbf{r}-\ell_{x} \mathbf{a}-\ell_{y} \mathbf{b}\right),
$$

where $\ell_{x} \in I_{x}$ means $M_{x}+1 \leq \ell_{x} \leq N_{x}-M_{x}$, and similarly for $\ell_{y} \in I_{y}$. The tile density $\rho_{\text {tile }}^{\mathrm{I}}(\mathbf{r})$ represents one unit cell, but does not have to be confined inside the rectangular boundaries of the cell; it can leak into neighboring cells, but the sum of these tile densities must exactly reproduce the bulk periodic density. Note that $\rho_{\text {tile }}^{\mathrm{I}}$ is net neutral, and since we assume inversion symmetry, we also require it to have a vanishing dipole moment.

The second term in Eq. (1) is a sum of four skin contributions, $\rho^{\mathrm{S}}=\rho^{\mathrm{T}}+\rho^{\mathrm{R}}+\rho^{\mathrm{B}}+\rho^{\mathrm{L}}$ (top, right, bottom, and left, respectively). Here, for example, the top skin contribution is

$$
\rho^{\mathrm{T}}(\mathbf{r})=\sum_{\ell_{x} \in I_{x}} \rho_{\mathrm{tile}}^{\mathrm{T}}\left(\mathbf{r}-\ell_{x} \mathbf{a}-N_{y} \mathbf{b}\right),
$$

where the "tile" $\rho_{\text {tile }}^{\mathrm{T}}(\mathbf{r})$ is only one unit cell wide, but comprises all of the $M_{y}$ vertically stacked cells in the top skin region. The density $\rho_{\text {tile }}^{\mathrm{T}}(\mathbf{r})$ must have the property that $\rho^{\mathrm{I}}(\mathbf{r})+\rho^{\mathrm{T}}(\mathbf{r})$ is identical to $\rho_{\text {flake }}(\mathbf{r})$ in the central region of the top edge. Similarly, in

$$
\rho^{\mathrm{R}}(\mathbf{r})=\sum_{\ell_{y} \in I_{y}} \rho_{\text {tile }}^{\mathrm{R}}\left(\mathbf{r}-\ell_{y} \mathbf{b}-N_{x} \mathbf{a}\right)
$$

the density $\rho_{\text {tile }}^{\mathrm{R}}(\mathbf{r})$ describes a region one cell high and $M_{x}$ cells wide at the right skin region. Since we are only interested in neutral edges, we will require all the tiles in the skin regions to be neutral, but they are generally not dipole-free.

The last term in Eq. (1) is a sum of contributions from the four corner regions,

$$
\rho^{\mathrm{C}}(\mathbf{r})=\rho_{\text {tile }}^{\mathrm{TR}}(\mathbf{r})+\rho_{\text {tile }}^{\mathrm{BR}}(\mathbf{r})+\rho_{\text {tile }}^{\mathrm{BL}}(\mathbf{r})+\rho_{\text {tile }}^{\mathrm{TL}}(\mathbf{r}),
$$


where each of these tiles is a larger one covering an entire corner region comprised of $M_{x} \times M_{y}$ unit cells. These corner tile densities need to make up for whatever charge density is missing after accounting for interior and skin contributions. For example, the top-right tile charge density is

$$
\rho_{\text {tile }}^{\mathrm{TR}}(\mathbf{r})=\rho_{\text {flake }}(\mathbf{r})-\rho^{\mathrm{I}}(\mathbf{r})-\rho^{\mathrm{T}}(\mathbf{r})-\rho^{\mathrm{R}}(\mathbf{r})
$$

restricted to the vicinity of this corner.

We now focus on the top-right corner, and let $Q_{\mathrm{c}}$ be the macroscopic charge of this corner, defined as the integral of a smoothened charge density over the corner region (see also Sec. IV A). This is given by

$$
Q_{\mathrm{c}}=\frac{1}{a b} q_{x y}^{\mathrm{I}}+\frac{1}{a} d_{x}^{\mathrm{T}}+\frac{1}{b} d_{y}^{\mathrm{R}}+Q^{\mathrm{TR}}
$$

where

$$
\begin{aligned}
q_{x y}^{\mathrm{I}} & =\int x y \rho_{\mathrm{tile}}^{\mathrm{I}}(\mathbf{r}) d^{2} r, \\
d_{x}^{\mathrm{T}} & =\int x \rho_{\mathrm{tile}}^{\mathrm{T}}(\mathbf{r}) d^{2} r, \\
d_{y}^{\mathrm{R}} & =\int y \rho_{\mathrm{tile}}^{\mathrm{R}}(\mathbf{r}) d^{2} r, \\
Q^{\mathrm{TR}} & =\int \rho_{\mathrm{tile}}^{\mathrm{TR}}(\mathbf{r}) d^{2} r .
\end{aligned}
$$

Working from right to left in Eq. (7), the contribution of $Q^{\mathrm{TR}}$ is obvious. The contribution from the right-edge tiles is that of a 1D chain of entities of dipole moment $d_{y}^{\mathrm{R}}$, Eq. (10), with density $1 / b$; this has $1 \mathrm{D}$ polarization $d_{y}^{\mathrm{R}} / b$, and thus contributes a bound end charge of that magnitude to the top end of the chain. The same applies to the $1 \mathrm{D}$ chain of $d_{x}^{\mathrm{T}}$ dipoles of density $1 / a$ at the top edge via Eq. (9). Finally, the superposition with density $1 / a b$ of identical, neutral, dipole-free quadrupoles $q_{x y}^{\mathrm{I}}$, Eq. (11), produces no macroscopic edge charge, but it does generate four macroscopic corner charges: $+q_{x y}^{\mathrm{I}} / a b$ at TR and BL, and $-q_{x y}^{\mathrm{I}} / a b$ at TL and BR. Combining all the contributions at the TR corner coming from Eqs. (8-11) results in Eq. (7), which will serve as an important basis for the remainder of this work.

Equation (7) is claimed to hold in the thermodynamic limit, but we expect rapid convergence with system size. The ideal situation occurs when the tile densities all have finite support, each vanishing outside its own local region. In that case, the $2 \mathrm{D}$ periodicity relating interior tiles and the $1 \mathrm{D}$ periodicity relating edge tiles guarantees that the coarse-grained charge density $\bar{\rho}(\mathbf{r})$, obtained using the sliding window average to be described in Sec. IV A, vanishes except near the corners as soon as $M_{x}$ and $M_{y}$ are large enough. The corner charge obtained by integrating $\bar{\rho}(\mathbf{r})$ over one of the corner regions then remains unchanged by any further increase of $M_{x}$ or $M_{y}$, so that perfect convergence to the thermodynamic limit is already achieved for modest values of $M_{x}$ and $M_{y}$. In practice the tile densities have exponential tails, in which case we expect exponential convergence with sample size, an expectation that is confirmed in the results to be presented below.

For future reference, it is useful to introduce the interior quadrupole density

$$
\mathcal{Q}_{x y}^{\mathrm{I}}=\frac{1}{a b} q_{x y}^{\mathrm{I}}
$$

and edge dipole densities

$$
\mathcal{P}_{x}^{\mathrm{T}}=\frac{1}{a} d_{x}^{\mathrm{T}}
$$

for the top edge and similarly for the other three edges. In this language, the top-right corner charge is

$$
Q_{\mathrm{c}}=\mathcal{Q}_{x y}^{\mathrm{I}}+\mathcal{P}_{x}^{\mathrm{T}}+\mathcal{P}_{y}^{\mathrm{R}}+Q^{\mathrm{TR}} .
$$

All quantities in Eq. (14) have units of charge $e$.

We emphasize that other definitions of edge polarizations are possible. First, the definitions of the bulk quadrupole density and surface dipole densities may differ from one formulation to another, and even within our approach, where it can depend on the choice of tile. Second, we would also be free to define

$$
\left.\begin{array}{l}
\overline{\mathcal{P}}_{x}^{\mathrm{T}}=\mathcal{P}_{x}^{\mathrm{T}}+\frac{1}{2} \mathcal{Q}_{x y}^{\mathrm{I}} \\
\overline{\mathcal{P}}_{y}^{\mathrm{R}}=\mathcal{P}_{y}^{\mathrm{R}}+\frac{1}{2} \mathcal{Q}_{x y}^{\mathrm{I}}
\end{array}\right\} \quad Q_{\mathrm{c}}=\overline{\mathcal{P}}_{x}^{\mathrm{T}}+\overline{\mathcal{P}}_{y}^{\mathrm{R}}
$$

or

$$
\left.\begin{array}{l}
\overline{\overline{\mathcal{P}}}_{x}^{\mathrm{T}}=\mathcal{P}_{x}^{\mathrm{T}}+\mathcal{Q}_{x y}^{\mathrm{I}} \\
\overline{\overline{\mathcal{P}}}_{y}^{\mathrm{R}}=\mathcal{P}_{y}^{\mathrm{R}}+\mathcal{Q}_{x y}^{\mathrm{I}}
\end{array}\right\} \quad Q_{\mathrm{c}}=\overline{\overline{\mathcal{P}}}_{x}^{\mathrm{T}}+\overline{\overline{\mathcal{P}}}_{y}^{\mathrm{R}}-\mathcal{Q}_{x y}^{\mathrm{I}}
$$

(written here for $Q^{\mathrm{TR}}=0$ ) in the spirit of some previous works $[4,5,15]$. Because we have concluded that the edge polarization is not a physical observable, we do not think that any one of these definitions is "more correct" than another. ${ }^{1}$ The reader is encouraged to beware of different definitions of these quantities when comparing papers from the literature.

\section{B. System of quantized charges}

We now assume that the charge density of the crystal is composed of quantized charges in multiples of $e$. This could be the fictitious world of integer point "ions" and integer point "electrons," but we will focus below on the case that the electrons are represented by WFs, each carrying charge $-e$ and exponentially localized in the vicinity of its WF center. The bulk tile $\rho_{\text {tile }}^{\mathrm{I}}(\mathbf{r})$ is

\footnotetext{
1 Note, however, that the formulation of Eq. (15) has the advantage of being be easily generalized to treat corners subtending angles other than $90^{\circ}$, as shown in Ref. [15].
} 
then constructed by choosing a set of representative ions and WFs to include in the home cell.

The dipole moment of this interior tile is

$$
d_{\mu}^{\mathrm{I}}=\int r_{\mu} \rho_{\mathrm{tile}}^{\mathrm{I}}(\mathbf{r}) d^{2} r
$$

Because we assumed inversion symmetry, the formal polarization, expressed in reduced units $p_{x}=d_{x}^{\mathrm{I}} / a e$, $p_{y}=d_{y}^{\mathrm{I}} / b e$, must map to itself, modulo integers, under inversion. There are four possible cases in which $\left(p_{x}, p_{y}\right)$ is either $(0,0),\left(0, \frac{1}{2}\right),\left(\frac{1}{2}, 0\right)$, or $\left(\frac{1}{2}, \frac{1}{2}\right)$, modulo integers. Only the first is fully nonpolar. The other three cases are somewhat trickier to handle, and for these we adopt a split-basis convention [24]. That is, we split one or more ions into several equal pieces, assigning these to unit cells in such a way that the home cell is dipole-free. For example, suppose there is one $+e$ ion at $(0,0)$ and one Wannier center at $(a / 2, b / 2)$, which would give $\mathbf{p}=\left(-\frac{1}{2},-\frac{1}{2}\right)$. In this case we could choose the home tile to consist of the WF density plus point ions of charge $+e / 4$ at $(0,0)$, $(a, 0),(0, b)$, and $(a, b)$, making for a dipole-free home cell. In this way, we will always arrange for $\rho_{\text {tile }}^{\mathrm{I}}$ to have zero dipole moment as well as zero net charge.

We also want to restrict ourselves to neutral edges, since otherwise the definition of a corner charge is problematic. For the $(0,0)$ case the edges are naturally neutral, and the edge tile, say at the top, just consists of some overall-neutral left-over set of ions and WFs. For the other cases, some edges are not naturally neutral, but they can always be made so by a period-doubling (or, for three-fold symmetries, period-tripling) edge reconstruction. We shall require that this has always been done. Since the (possibly split-basis-containing) bulk tiles are dipole-free by construction, the (possibly enlarged) edge tiles may also contain some fractional ionic charges, but they will always be neutral overall.

We note in passing that a similar split-basis approach was recently used to derive formulas for the quadrupole moment and corner charge [13]. The authors pointed out the gauge dependence of the quadrupole moment, but observed that it can be removed when the system has a $C_{n}$ rotational symmetry $(n=3,4,6)$. Mapping to a picture in which electrons are represented by point charges located at Wannier centers, they construct a charge-neutral and polarization-free basis by an appropriate assignment of Wannier centers to Wyckoff positions, an approach that is quite similar in spirit to our tiling decomposition. The method was implemented for a variety of model geometries in subsequent work [16]. However, these papers did not address the nonquantized corner charge that can appear when the $C_{n}$ symmetries are absent.

\section{Wannier representation and choice of home cell}

We now explicitly require that our 2D insulator must have a vanishing Chern number, since otherwise the presence of gapless edge channels would give rise to metallic boundaries, and there would be a topological obstruction to the construction of bulk WFs spanning the occupied bands.

Regarding the ionic charges, let the $i$ 'th ion in the home cell $\mathbf{R}=\mathbf{0}$ be located at $\boldsymbol{\tau}_{i}$ and carry charge $Z_{i} e$. Each ionic site $\boldsymbol{\tau}_{i}$ either sits on one of the four inversion centers in the unit cell, or they appear in pairs symmetrically arranged around an inversion center.

As for the electrons, we assume that a smooth and periodic bulk gauge has been chosen for the wave functions $\left|\psi_{n \mathbf{k}}\right\rangle$ of the $n=\{1, \ldots, J\}$ occupied bands, and that this gauge also respects the inversion symmetry. The WFs constructed from these bands have centers

$$
\overline{\mathbf{r}}_{\mathbf{R} n}=\langle\mathbf{R} n|\mathbf{r}| \mathbf{R} n\rangle=\mathbf{R}+\overline{\mathbf{r}}_{n} .
$$

Since the gauge respects inversion symmetry, the $\overline{\mathbf{r}}_{n}$ are also located on inversion centers or are symmetrically disposed about them in pairs. When we consider our flake, we assume that the WFs of the flake become identical to these bulk WFs deep in the interior of the flake, so that the home-cell charge distribution $\rho_{\text {tile }}^{\mathrm{I}}$ is just built from these ions and WFs. As discussed in the previous section, this tile will always be dipole-free, even if it requires splitting some ionic charges.

It may be useful to introduce a set of reference $W F$ center positions as follows. For each WF $|\mathbf{0} n\rangle$ that sits on one of the inversion centers, we define $\mathfrak{t}_{n}$ to be the location of that inversion center (i.e., equal to $\overline{\mathbf{r}}_{n}$ ); and for every pair of WF centers symmetrically disposed about one of the inversion centers, we again assign $\mathfrak{t}_{n}$ for each of them to be at that inversion center. Then the interior tile charge density

$$
\rho_{\text {tile }}^{\mathrm{I}}(\mathbf{r})=e \sum_{i} Z_{i} \delta^{2}\left(\mathbf{r}-\boldsymbol{\tau}_{i}\right)-e \sum_{n}|\langle\mathbf{r} \mid \mathbf{0} n\rangle|^{2}
$$

can be written as

$$
\rho_{\text {tile }}^{\mathrm{I}}(\mathbf{r})=\rho_{\text {tile }}^{\text {ion }}(\mathbf{r})+\rho_{\text {tile }}^{\text {el }}(\mathbf{r})
$$

where

$$
\rho_{\text {tile }}^{\text {ion }}(\mathbf{r})=e \sum_{i} Z_{i} \delta^{2}\left(\mathbf{r}-\boldsymbol{\tau}_{i}\right)-e \sum_{n} \delta^{2}\left(\mathbf{r}-\mathfrak{t}_{n}\right)
$$

and

$$
\rho_{\text {tile }}^{\mathrm{el}}(\mathbf{r})=-e \sum_{n}\left[|\langle\mathbf{r} \mid \mathbf{0} n\rangle|^{2}-\delta^{2}\left(\mathbf{r}-\mathbf{t}_{n}\right)\right] .
$$

The advantage of this formulation is that $\rho_{\text {tile }}^{\text {ion }}$ is a purely classical point charge distribution that is gaugeindependent, ${ }^{2}$ while all of the electronic gauge dependence is carried by $\rho_{\text {tile }}^{\mathrm{el}}$.

\footnotetext{
2 To be clear, there are "large" or "radical" gauge transformations that shift one or more WF centers by a lattice vector, and "small" or "progressive" ones that can be smoothly connected to the identity gauge transformation. We assume that the former are built into the definition of the contents of the unit cell, so at this point when we speak of gauge transformations, we mean progressive ones only.
} 


\section{Wannier quadrupoles and dipoles}

We are now ready to put it all together. The ingredients needed to compute the upper-right corner charge of Eq. (7) are given as follows. The bulk quadrupole is

$$
\begin{aligned}
q_{x y}^{\mathrm{I}} & =e \sum_{i}^{\mathrm{I}} Z_{i} \tau_{i x} \tau_{i y}-e \sum_{n}^{\mathrm{I}}\langle\mathbf{0} n|x y| \mathbf{0} n\rangle \\
& =q_{x y}^{\mathrm{ion}}+q_{x y}^{\mathrm{el}},
\end{aligned}
$$

where the sums are over the contents of the interior (I) tile, and $q_{x y}^{\text {ion }}$ and $q_{x y}^{\mathrm{el}}$ are the quadrupoles of the distributions in Eqs. (21-22), i.e.,

$$
\begin{aligned}
q_{x y}^{\text {ion }} & =e \sum_{i}^{\mathrm{I}} Z_{i} \tau_{i x} \tau_{i y}-e \sum_{n}^{\mathrm{I}} \mathfrak{t}_{n x} \mathfrak{t}_{n y}, \\
q_{x y}^{\mathrm{el}} & =-e \sum_{n}^{\mathrm{I}}\left[\langle\mathbf{0} n|x y| \mathbf{0} n\rangle-\mathfrak{t}_{n x} \mathfrak{t}_{n y}\right] .
\end{aligned}
$$

The $x$ dipole of a top-edge tile is

$$
d_{x}^{\mathrm{T}}=e \sum_{i}^{\mathrm{T}} Z_{i} \tau_{i x}-e \sum_{n}^{\mathrm{T}}\langle 0 n|x| 0 n\rangle,
$$

where this time the sum is over the contents of the top edge tile, and $\left|l_{x} n\right\rangle$ denotes a WF belonging to the $l_{x}^{\text {th }}$ tile along the edge. Similarly,

$$
d_{y}^{\mathrm{R}}=e \sum_{i}^{\mathrm{R}} Z_{i} \tau_{i y}-e \sum_{n}^{\mathrm{R}}\langle 0 n|y| 0 n\rangle,
$$

where the ket notation is $\left|l_{y} n\right\rangle$. Finally,

$$
Q^{\mathrm{TR}}=e \sum_{i}^{\mathrm{TR}} Z_{i}-e N^{\mathrm{TR}}
$$

where $N^{\text {TR }}$ is the number of WFs associated with the top-right corner tile. Inserting Eqs. (23-28) into Eq. (7) yields the desired expression for the top-right corner charge.

If we are only interested in the corner charge $\bmod e$, then no electronic solution is needed for the TR region; $Q^{\mathrm{TR}}$ vanishes mod $e$ if fractional ionic charges $Z_{i}$ are absent, and are easily determined if they are present. Thus, $Q_{\mathrm{c}}$ can be determined mod $e$ using only calculations on two infinite ribbons and a knowledge of the ionic arrangement at the corner. If we want to know $Q_{\mathrm{c}}$ fully, not just $\bmod e$, then we also need enough information about the electronic structure of the flake to decide the number $N^{\mathrm{TR}}$ of occupied WFs in the corner tile.

\section{GAUGE DEPENDENCE OF INTERIOR QUADRUPOLES AND EDGE DIPOLES}

In Secs. II C and II D we assumed some definite choice of WFs providing a representation of the occupied electronic states of the flake. Specifically, the set of all bulk,

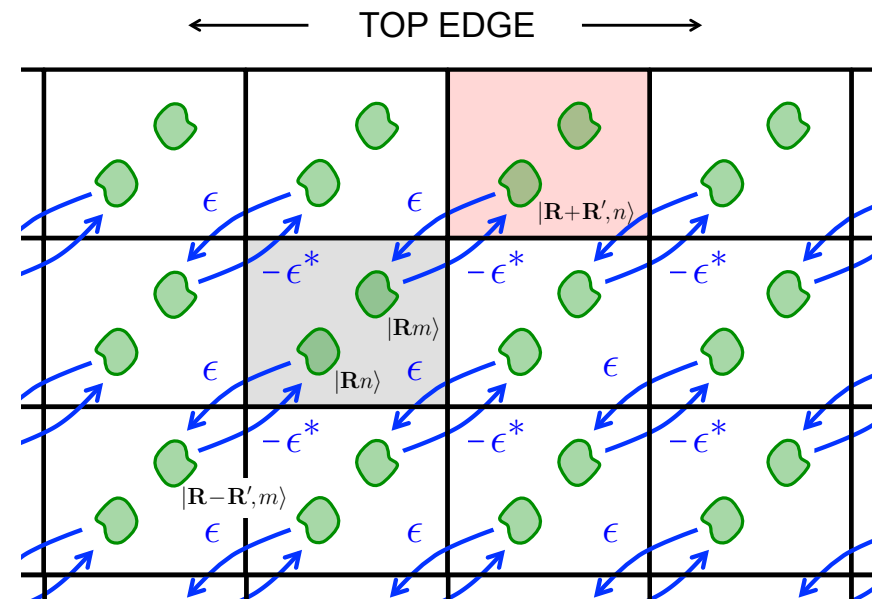

FIG. 2. Sketch of top edge of sample, showing mixing of Wannier functions under the infinitesimal gauge transformation of Eq. (30). Gray cell is an interior cell whose dipole moment is unchanged due to cancellation of the $\epsilon$ contribution from above and the $-\epsilon^{*}$ one from below; pink cell is a top skin cell whose dipole does shift as a result of the unbalanced $-\epsilon^{*}$ contribution from below.

skin, and corner WFs must be orthonormal and must exactly span the occupied band subspace of the flake. We refer to any particular choice of WFs as a "choice of gauge." This choice is not unique, so it is important to discuss the gauge dependence of quantities such as $q_{x y}^{\text {el }}$ and $d_{x}^{\mathrm{T}}$ of Eqs. (25-26).

A general gauge transformation corresponds to a unitary mixing of the WFs according to

$$
\left|\mathbf{R}_{1} n_{1}\right\rangle_{\text {new }}=\sum_{\mathbf{R}_{2} n_{2}} U_{\mathbf{R}_{2} n_{2}, \mathbf{R}_{1} n_{1}}\left|\mathbf{R}_{2} n_{2}\right\rangle,
$$

where $U$ is unitary. For our purposes, it is sufficient to consider the transformation properties under infinitesimal unitary transformations, since finite gauge transformations can always be built up by using these as generators. $^{3}$ The general form of an infinitesimal unitary operator is $U=e^{A}=1+A$ for infinitesimal antihermitian $A$. In the bulk part of the flake, we want the WFs to retain the property of being periodic images of each other, so we require that $A$ be lattice-periodic, i.e., $A_{\mathbf{R}_{1} n_{1}, \mathbf{R}_{2} n_{2}}=A_{\mathbf{R}_{1}+\mathbf{R}^{\prime}, n_{1}, \mathbf{R}_{2}+\mathbf{R}^{\prime}, n_{2}}$. We further specialize to the case that $A$ specifies a mixing of amplitude $\epsilon$ between WF $n_{1}=m$ in cell $\mathbf{R}_{1}=\mathbf{R}$ and WF $n_{2}=n$ in cell $\mathbf{R}_{2}=\mathbf{R}+\mathbf{R}^{\prime}$, since more general gauge transformations can again be built up from elementary ones such as this.

The first-order changes in the WFs in tile $\mathbf{R}$ are then

\footnotetext{
3 Strictly speaking, this only applies to "small" or "progressive" gauge transformations, i.e., those that can be continuously deformed to the identity. "Large" or "radical" gauge transformations that shift some WFs into a neighboring cell are also possible, but these would correspond to a different choice of tiling.
} 
given by

$$
\begin{aligned}
\delta|\mathbf{R} m\rangle & =\epsilon\left|\mathbf{R}+\mathbf{R}^{\prime}, n\right\rangle, \\
\delta|\mathbf{R} n\rangle & =-\epsilon^{*}\left|\mathbf{R}-\mathbf{R}^{\prime}, m\right\rangle,
\end{aligned}
$$

with other WFs in the cell being unaffected. The mixing pattern is illustrated in Fig. 2. For an arbitrary singleparticle operator $\mathcal{O}$, the change of its trace over the WFs in cell $\mathbf{R}$ is given by

$$
\begin{aligned}
\delta\langle\mathcal{O}\rangle_{\text {tile }}= & \delta\langle\mathbf{R} m|\mathcal{O}| \mathbf{R} m\rangle+\delta\langle\mathbf{R} n|\mathcal{O}| \mathbf{R} n\rangle \\
= & 2 \operatorname{Re}\left[\epsilon\left\langle\mathbf{R} m|\mathcal{O}| \mathbf{R}+\mathbf{R}^{\prime}, n\right\rangle\right. \\
& \left.\quad-\epsilon\left\langle\mathbf{R}-\mathbf{R}^{\prime}, m|\mathcal{O}| \mathbf{R} n\right\rangle\right] \\
= & 2 \operatorname{Re}\left[\epsilon\left\langle\mathbf{R} m\left|\left[\mathcal{O}, T_{\mathbf{R}^{\prime}}\right]\right| \mathbf{R} n\right\rangle\right]
\end{aligned}
$$

where $T_{\mathbf{R}}$ is the operator that translates by lattice vector R. For a lattice-periodic operator such as the bulk Hamiltonian, the commutator in Eq. (31) vanishes, and the density of $\mathcal{O}$ per unit cell is gauge-invariant.

However, we are interested in dipoles and quadrupoles, and for these cases we have that $\left[x, T_{\mathbf{R}}\right]=R_{x} T_{\mathbf{R}}$, $\left[y, T_{\mathbf{R}}\right]=R_{y} T_{\mathbf{R}}$, and $\left[x y, T_{\mathbf{R}}\right]=\left(y R_{x}+x R_{y}+R_{x} R_{y}\right) T_{\mathbf{R}}$. Using the orthogonality of the WFs, $\left\langle\mathbf{R} m \mid \mathbf{R}+\mathbf{R}^{\prime}, n\right\rangle=$ $\delta_{\mathbf{0 R}} \delta_{m n}$, it follows that

$$
\begin{aligned}
\delta\langle x\rangle_{\mathrm{tile}} & =\delta\langle y\rangle_{\mathrm{tile}}=0 \\
\delta\langle x y\rangle_{\mathrm{tile}} & =2 R_{x}^{\prime} \operatorname{Re}\left[\epsilon Y_{m n}^{\mathbf{R}^{\prime}}\right]+2 R_{y}^{\prime} \operatorname{Re}\left[\epsilon X_{m n}^{\mathbf{R}^{\prime}}\right],
\end{aligned}
$$

where

$$
\begin{aligned}
X_{m n}^{\mathbf{R}} & =\langle\mathbf{0} m|x| \mathbf{R} n\rangle, \\
Y_{m n}^{\mathbf{R}} & =\langle\mathbf{0} m|y| \mathbf{R} n\rangle .
\end{aligned}
$$

Equation (32) confirms that the dipole moment of the Wannier charge distribution in a bulk tile is gaugeinvariant, as expected since it corresponds to the electric polarization. Another way to see this is to compute the shifts of the Wannier centers $\bar{x}_{\mathbf{R} m}=\langle\mathbf{R} m|x| \mathbf{R} m\rangle=$ $R_{x}+\bar{x}_{m} ;$ using the same methods, we obtain

$$
\delta \bar{x}_{m}=-\delta \bar{x}_{n}=2 \operatorname{Re}\left[\epsilon X_{m n}^{\mathbf{R}^{\prime}}\right],
$$

and similarly for $\delta \bar{y}$. The two WF centers thus shift by equal distances but in opposite directions, preserving the overall cell dipole.

However, the gauge invariance of the dipole does not extend to the quadrupole. From Eq. (12), (23), and (33) we obtain

$$
\delta \mathcal{Q}_{x y}^{\mathrm{I}}=-\frac{2 e}{a b}\left(R_{x}^{\prime} \operatorname{Re}\left[\epsilon Y_{m n}^{\mathbf{R}^{\prime}}\right]+R_{y}^{\prime} \operatorname{Re}\left[\epsilon X_{m n}^{\mathbf{R}^{\prime}}\right]\right) .
$$

This shows that the bulk quadrupole moment of an interior tile is not a gauge-invariant quantity. In particular, this suggests that it is not a physical observable.

Now let us concentrate our attention on the skin region, specifically at the top edge of the flake. The quadrupoles in this region are of no interest, since the area of the skin region becomes negligible in the limit of a large flake. A gauge change that is restricted only to the skin region cannot change the dipole moment of an edge tile, by an argument similar to that leading to Eq. (32).

Surprisingly, though, the dipole of an edge tile can be modified by an interior gauge transformation. To see this, we return to Fig. 2 and discuss it in the context of Eq. (35). Note that Fig. 2 is drawn for the case that $\mathbf{R}^{\prime}=\ell_{x} \mathbf{a}+\ell_{y} \mathbf{b}$ with $\ell_{x}=\ell_{y}=1$, and for simplicity we assume that the skin tile is only one unit cell thick. In this case, each skin tile "donates" a contribution $-2 e \operatorname{Re}\left[\epsilon X_{m n}^{\mathbf{R}^{\prime}}\right]$ to one of the top-most interior tiles below it, as illustrated by the blue arrow marked $\epsilon^{*}$ pointing from $\left|\mathbf{R}+\mathbf{R}^{\prime}, n\right\rangle$ in the pink skin cell to $|\mathbf{R} m\rangle$ in the gray interior cell in Fig. 2. As a result, the shift of $\overline{\mathbf{r}}_{\mathbf{R}+\mathbf{R}^{\prime}, n}$ adds to the dipole of the pink edge tile by $2 e \operatorname{Re}\left[\epsilon X_{m n}^{\mathbf{R}^{\prime}}\right]$, and the shift of $\overline{\mathbf{r}}_{\mathbf{R} m}$ in the gray tile makes an equal and opposite contribution to the gray-tile dipole. However, there is no net change of the gray-tile dipole, since it receives a compensating donation marked by the $-\epsilon^{*}$ arrow from the deeper tile below it. By contrast, no such cancellation occurs for the pink tile, so there is a net change of its dipole, and a resulting change by $(2 e / a) \operatorname{Re}\left[\epsilon X_{m n}^{\mathbf{R}^{\prime}}\right]$ of the edge polarization $\mathcal{P}_{x}^{\mathrm{T}}$.

This result depends crucially on the choice of $\ell_{y}=1$, as in Fig. 2, for the relative lattice vector $\mathbf{R}^{\prime}$ involved in the unitary mixing. If $\ell_{y}=2$, then there are two uncompensated contributions to the edge tile instead of one, and if $\ell_{y}=-1$, then the transfer of dipole moment goes in the reverse direction. Overall, then, we find that $\delta d_{x}^{\mathrm{T}}=2 e \ell_{y} \operatorname{Re}\left[\epsilon X_{m n}^{\mathbf{R}^{\prime}}\right]$, and using Eqs. (13) and (26) together with $\ell_{y}=R_{y}^{\prime} / b$, and applying similar considerations to the right edge, we find that the bulk-gaugeinduced changes to the edge dipole densities are

$$
\begin{aligned}
\delta \mathcal{P}_{x}^{\mathrm{T}} & =\frac{2 e}{a b} R_{y}^{\prime} \operatorname{Re}\left[\epsilon X_{m n}^{\mathbf{R}^{\prime}}\right], \\
\delta \mathcal{P}_{y}^{\mathrm{R}} & =\frac{2 e}{a b} R_{x}^{\prime} \operatorname{Re}\left[\epsilon Y_{m n}^{\mathbf{R}^{\prime}}\right] .
\end{aligned}
$$

Finally, as for the top-right corner tile, neither its quadrupole nor its dipole can contribute to the macroscopic corner charge. Moreover, its net charge density, given by Eq. (28), is obviously gauge-invariant, so that $\delta Q^{\mathrm{TR}}=0$.

Combining these contributions to Eq. (14), we find that the contributions from Eqs. (37) and (38) exactly cancel the one from Eq. (36), so that

$$
\delta Q_{\mathrm{c}}=\delta \mathcal{Q}_{x y}^{\mathrm{I}}+\delta \mathcal{P}_{x}^{\mathrm{T}}+\delta \mathcal{P}_{y}^{\mathrm{R}}=0
$$

In other words, the bulk quadrupole density and edge dipole densities are individually gauge-dependent, but their sum is gauge-invariant and describes a physical observable, the corner charge. This is a major result of our work.

A crucial consequence of this result is that the corner charge $Q_{\mathrm{c}}$ can be obtained modulo $e$ from independent calculations of $\mathcal{Q}_{x y}^{\mathrm{I}}, \mathcal{P}_{x}^{\mathrm{T}}$, and $\mathcal{P}_{y}^{\mathrm{R}}$, but only if all three 
contributions are computed using the same bulk gauge. For example, by studying ribbons that are finite in $y$ and infinite along $x$, we can compute $\mathcal{Q}_{x y}^{\mathrm{I}}$ from the charge density of a deep interior tile, and $\mathcal{P}_{x}^{\mathrm{T}}$ from that of an edge tile, and we can get $\mathcal{P}_{y}^{\mathrm{R}}$ in a similar way from a ribbon that is finite in $x$ instead. However, unless we insist that the bulk gauge is the same, we cannot use Eq. (14) to compute the corner charge by summing these ingredients. For example, if one obtains $\mathcal{P}_{x}^{\mathrm{T}}$ from a $y$-finite ribbon Wannierized along $\hat{\mathbf{y}}$ and $\mathcal{P}_{y}^{\mathrm{R}}$ from an $x$-finite ribbon Wannierized along $\hat{\mathbf{x}}$ as described in Sec. IV B 2 below, then in general the gauges are not consistent, and the $\operatorname{sum} \mathcal{P}_{x}^{\mathrm{T}}+\mathcal{P}_{y}^{\mathrm{R}}$ is not meaningful. (An exception to this rule will be discussed in Sec. VA.)

While preparing this manuscript, we became aware of a recent work that proposes a "thermodynamic" definition of gauge-invariant electric quadrupole moments [26]. However, the underlying formulation of this approach is very different from ours; it aims to describe local polarizations induced by slow spatial variations of a bulk Hamiltonian, and makes no claim to predict surface or corner properties except in the case of quantizing symmetries. The two approaches are thus complementary, and investigations into the relations between them may be a fruitful avenue for future investigation.

\section{METHODS}

In this work, we use simple tight-binding models for the purpose of implementing our formalism and testing its predictions. These will be introduced in detail in Sec. V. Each model is specified by providing the location of each basis orbital $\left|\varphi_{\mathbf{0} i}\right\rangle$ in the rectangular $a \times b$ home unit cell, implying periodic images $\left|\varphi_{\mathbf{R} i}\right\rangle=T_{\mathbf{R}}\left|\varphi_{\mathbf{0} i}\right\rangle$ in other cells. The on-site energy of each basis orbital, and the hoppings connecting near-neighbor orbitals, are also specified. The position operator is assumed to be diagonal in the tightbinding basis, $\left\langle\varphi_{\mathbf{R} i}|\mathbf{r}| \varphi_{\mathbf{R}^{\prime} j}\right\rangle=\left(\mathbf{R}+\boldsymbol{\tau}_{i}\right) \delta_{\mathbf{R}, \mathbf{R}^{\prime}} \delta_{i j}$, with $\boldsymbol{\tau}_{i}$ denoting the location of the $i$ th basis function in the home cell. We treat the charge density of each basis orbital as a Dirac delta function, $\left|\left\langle\mathbf{r} \mid \varphi_{\mathbf{R} i}\right\rangle\right|^{2}=\delta^{2}\left(\mathbf{r}-\mathbf{R}-\boldsymbol{\tau}_{i}\right)$, so that the basis functions themselves have zero spread. Positive ionic charges are assigned to all of the tightbinding sites to neutralize the unit cell. The electronic Hamiltonian for bulk, ribbon, and flake geometries is constructed and solved using the PYTHTB code package [27].

\section{A. Corner charge and macroscopic averaging}

To calculate the corner charge directly, we construct a rectangular flake consisting of $N_{x} \times N_{y}$ unit cells, and obtain the total charge $q_{\mathbf{R} i}$ (ionic plus electronic) on every site. Since we associate the electronic charge to delta functions on the sites, the total charge density takes the

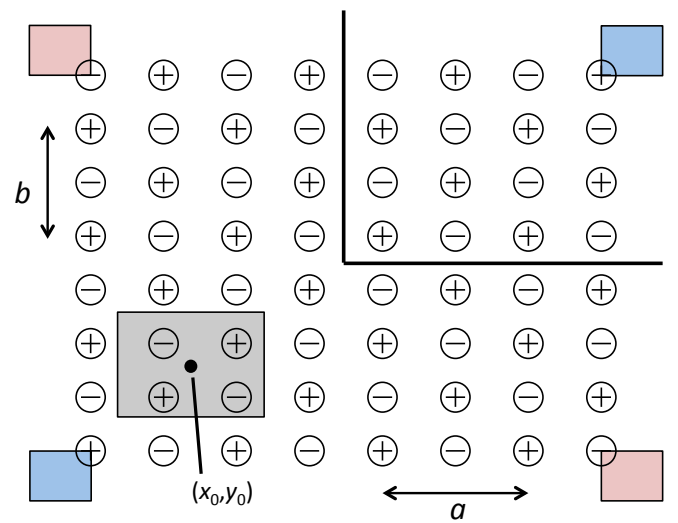

FIG. 3. Sketch of sliding-window approach for obtaining macroscopic corner charges. The macroscopically averaged charge density $\bar{\rho}\left(\mathbf{r}_{0}\right)$ is defined as the average of $\rho(\mathbf{r})$ over a rectangular cell centered at $\mathbf{r}_{0}=\left(x_{0}, y_{0}\right)$. For this model of $\pm e$ point charges, $\bar{\rho}$ vanishes except in the blue and pink rectangles, where it takes values $+e / a b$ and $-e / a b$ respectively. Integration of the charge in one of these colored rectangles yields the corresponding macroscopic corner charge.

form

$$
\rho(\mathbf{r})=\sum_{\mathbf{R} i} q_{\mathbf{R} i} \delta^{2}\left(\mathbf{r}-\mathbf{R}-\boldsymbol{\tau}_{i}\right) .
$$

The macroscopic corner charge is determined by first applying a smoothening procedure, since simple sums of individual charges are not convergent. For this purpose we adopt the sliding window average approach [3, 28], in which a broadened charge density $\bar{\rho}(\mathbf{r})$ is obtained by convoluting $\rho(\mathbf{r})$ with a "window function"

$$
w(x, y)= \begin{cases}1 / a b & \text { if }|x|<a / 2,|y|<b / 2 \\ 0 & \text { otherwise }\end{cases}
$$

i.e.,

$$
\bar{\rho}\left(\mathbf{r}_{0}\right)=\int \rho\left(\mathbf{r}_{0}-\mathbf{r}^{\prime}\right) w\left(\mathbf{r}^{\prime}\right) d^{2} r^{\prime} .
$$

The advantage of this procedure is that $\bar{\rho}(\mathbf{r})$ is guaranteed to vanish in the bulk-like regions of the sample as a result of the charge neutrality of the bulk unit cell. We also assume that the bulk has been terminated in such a way as to yield neutral edges, as described in Sec. II B, so that $\bar{\rho}(\mathbf{r})$ vanishes there as well. The corner charge is then obtained by integrating the smoothened charge density over the corner of interest.

The application of the above macroscopic averaging procedure to a simple checkerboard arrangement of $\pm e$ point charges is illustrated in Fig. 3. The range of the window function centered on position $\mathbf{r}_{0}=\left(x_{0}, y_{0}\right)$ is shown by the gray rectangle. As one slides this window around on the sample, the charge contained in it vanishes except when $\mathbf{r}_{0}$ falls in the rectangular $a / 2 \times b / 2$ regions, where $\pm \bar{\rho}=e / a b$ in the blue and red rectangles respectively. Thus, the macroscopic edge charges are zero for 
this model crystal, and the macroscopic corner charges are $\pm e / 4$, with the positive charges at top right and bottom left.

Other coarse-graining approaches will lead to the same result. For example, Gaussian broadening can also be used, but then a careful treatment of the two limits $\max (a, b) \ll \sigma \ll \min \left(L_{x} a, L_{y} b\right)$ has to be enforced, where $\sigma$ is the Gaussian width. The sliding window approach avoids such complications. Note, however, that a simple summation of the charges in a quadrant of the flake, as shown by the heavy black lines, does not yield the correct corner charge. For the quadrant shown, it yields zero; if the quadrant contained $5 \times 5$ instead of $4 \times 4$ cells, it would yield $+e$. Neither value is correct.

The technical implementation of the sliding window averaging procedure is as follows. We can write

$$
Q_{\mathrm{c}}=\int d^{2} r \Lambda(\mathbf{r}) \bar{\rho}(\mathbf{r})
$$

where $\Lambda(\mathbf{r})=1$ in an upper-right-hand region $x>x_{0}$ and $y>y_{0}$ and zero otherwise for appropriately chosen $x_{0}$ and $y_{0}$. In the language of function spaces this is the inner product $\Lambda \circ \bar{\rho}$, while $\bar{\rho}$ is the convolution $\bar{\rho}=\rho * w$; noting that $w(\mathbf{r})=w(-\mathbf{r})$, this is equivalent to $Q_{\mathrm{c}}=$ $W \circ \rho$ with $W=\Lambda * w$. Thus, in practice we compute the macroscopic corner charge as

$$
Q_{\mathrm{c}}=\int d^{2} r W(\mathbf{r}) \rho(\mathbf{r}),
$$

with $W(x, y)=f_{a}\left(x-x_{0}\right) f_{b}\left(y-y_{0}\right)$ given by the product of two "ramp functions" defined as $f_{d}(u)=0$ for $u<-d / 2,1$ for $u>d / 2$, and $1 / 2+u / d$ in the interval $[-d / 2, d / 2]$. Note that Eq. (44) is not the same as the bare $Q_{\mathrm{c}}$ obtained by integrating $\rho(\mathbf{r})$ over a quadrant, i.e,

$$
Q_{\mathrm{c}}^{\mathrm{bare}}=\int d^{2} r \Lambda(\mathbf{r}) \rho(\mathbf{r})
$$

for $x_{0}$ and $y_{0}$ at the sample center. This definition of $Q_{c}$ was used in Refs. [5, 8, 17, and 18], and the difference with respect to the macroscopic $Q_{c}$ of Eqs. (43-44) will be discussed in Sec. V C.

\section{B. Wannier construction for ribbon models}

Our goal is to use our formalism to predict corner charges from edge polarizations and interior quadrupoles computed for $x$ - and $y$-finite ribbon models. For example, we cut from the infinite $2 \mathrm{D}$ bulk a ribbon that is finite and $N_{y}$ cells thick in the $y$ direction, but still infinite and periodic in the $x$ direction. In this case the wavevector $k_{x}$ is a good quantum number, and we obtain the Bloch states according to the eigenvalue equation

$$
H\left|\psi_{k_{x} n}\right\rangle=E_{k_{x} n}\left|\psi_{k_{x} n}\right\rangle .
$$

We are interested only in the occupied wavefunctions, so for consistency with the bulk which has $J$ occupied bands, we let $n$ run over $N_{y} J$ occupied ribbon bands at each $k_{x}$. We then need to construct a specific gauge for the WFs spanning the occupied states, and in the following we present three different strategies for doing so.

We first present, in Sec. IV B 1, a method based on projecting onto trial functions. As the same trial functions are used for both $x$-finite and $y$-finite ribbons, this yields a consistent gauge, allowing for a viable calculation of the corner charge.

Next, we discuss Wannier constructions based on assigning states to layers via a preliminary maximal localization in one direction, followed by maximal localization within each layer in the orthogonal direction. If the first step is taken in the transverse (finite) direction, it corresponds to the "hybrid Wannier" construction; the occupied subspace is represented in terms of states that are exponentially localized in the transverse direction, while remaining extended and labeled by wavevector in the longitudinal direction. However, we then follow by a second localization step to arrive at fully localized WFs. This "transverse-first" nested Wannier construction is described in Sec. IVB 2. We also consider the reverse order of operations, in which the preliminary localization is carried out in the extended direction; this "longitudinal-first" nested Wannier construction is described in Sec. IV B 3.

In Ref. [23], the transverse-first hybrid Wannier construction was applied to both the $x$-finite and $y$-finite ribbons. We emphasize that in general this does not produce the same gauge for the interior WFs of the two ribbons, and hence it cannot safely be used to predict the corner charge. This will later be demonstrated explicitly in Sec. V B. (Centrosymmetric models with a single occupied band and time-reversal symmetry provide an exception, as will be discussed in Sec. VA.) Instead, if either the $x$-first or $y$-first nested Wannier scheme is consistently adopted for both ribbons (transverse for one ribbon and longitudinal for the other), then we arrive at a second viable approach for computing the corner charge, as discussed later in Sec. VI.

In the following, we focus for concreteness on $y$-finite ribbons and discuss each of the WF construction schemes in this context.

\section{Projection-based Wannier construction}

One approach to the construction of a gauge, and one that automatically produces the same gauge for both ribbons, is to use the trial function projection method $[21,22]$. In this approach, one invents $J$ trial functions $\left|g_{n}\right\rangle$ in the home unit cell that are intended as a rough approximation to the desired bulk WFs, with $g_{\ell_{x} \ell_{y} n}(\mathbf{r})=g_{n}\left(\mathbf{r}-\ell_{x} \mathbf{a}-\ell_{y} \mathbf{b}\right)$ being their translational images. Then considering a $y$-finite ribbon, for example, we construct a set of ribbon trial functions by taking the $\left|g_{\ell_{x} \ell_{y} n}\right\rangle$ with $\ell_{x}$ running over all integers while $\ell_{y}$ runs 
over the $N_{y}$ layers in the ribbon, with possible additions or deletions in the skin region to match the expected occupation of edge and corner states (see, e.g., Sec. VC). The goal then is to construct a set of WFs $\left|w_{\ell_{x} \ell_{y} n}\right\rangle$ that look "as similar as possible" to these $\left|g_{\ell_{x} \ell_{y} n}\right\rangle$, while still being built only from occupied Bloch states.

This is most easily done by going to reciprocal space. Temporarily introducing the composite index $\alpha=\left(\ell_{y} n\right)$, we define trial Bloch functions

$$
\left|\tilde{g}_{k_{x} \alpha}\right\rangle=N_{x}^{-1 / 2} \sum_{\ell_{x}} e^{i k_{x} \ell_{x} a}\left|g_{\ell_{x} \alpha}\right\rangle
$$

and construct the overlap matrix

$$
B_{k_{x}, \alpha \beta}=\left\langle\psi_{k_{x} \alpha} \mid \tilde{g}_{k_{x} \beta}\right\rangle .
$$

If our choice of trial functions had been ideal in the sense that the $\left|\tilde{g}_{k_{x} \alpha}\right\rangle$ had spanned the occupied subspace at $k_{x}$, $B_{k_{x}}$ would be a unitary matrix. More generally, we find the unitary part $\mathcal{B}$ of the $B$ matrix by subjecting it to the singular value decomposition $B=V \Sigma W^{\dagger}(V$ and $W$ are unitary and $\Sigma$ is positive real diagonal), and choosing $\mathcal{B}=V W^{\dagger}$. We also monitor the singular values (diagonal elements of $\Sigma$ ); if any of them becomes much less than unity, this signals the need to choose a different set of trial functions.

We then construct mixtures of Bloch functions such that the resulting ones are maximally aligned to the $\left|\tilde{g}_{k_{x} \alpha}\right\rangle$ according to

$$
\left|h_{k_{x} \alpha}\right\rangle=\sum_{\beta} \mathcal{B}_{k_{x}, \beta \alpha}\left|\psi_{k_{x} \beta}\right\rangle .
$$

Restoring $\alpha=\left(\ell_{y} n\right)$, these $\left|h_{k_{x} \ell_{y} n}\right\rangle$ can be interpreted as hybrid Wannier functions, as they are exponentially localized in the finite direction while remaining extended and labeled by wavevector $k_{x}$ in the extended direction. From these, we can construct fully localized WFs by carrying out the Fourier transform

$$
\left|w_{\ell_{x} \ell_{y} n}\right\rangle=\frac{a}{2 \pi} \int d k_{x} e^{-i k_{x} \ell_{x} a}\left|h_{k_{x} \ell_{y} n}\right\rangle .
$$

In the deep interior of the ribbon, all of these WFs will be periodic images of those in neighboring cells.

We now pick the WFs associated with one central cell with labels $\left(\ell_{x} \ell_{y}\right)$ and sum the $\left\langle w_{\ell_{x} \ell_{y} n}|x y| w_{\ell_{x} \ell_{y} n}\right\rangle$ over $n$ to obtain the interior quadrupole $q_{x y}^{\mathrm{I}}$ via Eq. (23), where $|\mathbf{0} n\rangle$ in the notation of Eq. (23) is the same as $\left|w_{\ell_{x} \ell_{y} n}\right\rangle$ here. Similarly, we define the skin region at the top edge of the sample to consist of some number $M_{y}$ of the top-most layers. Since the dipole moments of these cells vanish exponentially with depth, a fairly small value of $M_{y}$ is typically sufficient. Then, the $x$ dipole moments $\left\langle w_{\ell_{x} \ell_{y} n}|x| w_{\ell_{x} \ell_{y} n}\right\rangle$ are summed to provide the needed contributions to the total dipole $d_{x}^{\mathrm{T}}$ of Eq. (26).

We emphasize that our projection procedure insures that if we start from the same set of trial functions, the gauges in the interior region are the same by construction for $y$-finite and $x$-finite ribbons. Thus, we should expect to find the same $\mathcal{Q}_{x y}^{\mathrm{I}}$ for both ribbons; we confirm this below. Moreover, with the results of both ribbon calculations in hand, we are assured that the set of quantities $\mathcal{P}_{x}^{\mathrm{T}}, \mathcal{P}_{y}^{\mathrm{R}}$, and $\mathcal{Q}_{x y}^{\mathrm{I}}$ have been computed in a common gauge, and can confidently be combined as in Eq. (14) to predict the corner charge.

\section{Transverse-first nested Wannier construction}

Let us now discuss an alternative Wannier construction procedure that does not require choosing a set of trial functions. Again taking a $y$-finite ribbon and noting that matrix elements of the position operator $\hat{y}$ are well defined, it is straightforward to obtain the matrix

$$
Y_{k_{x}, m n}=\left\langle\psi_{k_{x} m}|y| \psi_{k_{x} n}\right\rangle,
$$

where $m$ and $n$ run over the $N_{y} J$ occupied bands of the ribbon at a given $k_{x}$, and to diagonalize it,

$$
\sum_{n} Y_{k_{x}, m n} \xi_{k_{x} \alpha, n}=\bar{y}_{k_{x} \alpha} \xi_{k_{x} \alpha, m},
$$

where $\alpha=\left\{1, \ldots, N_{y} J\right\}$ now labels the eigenvalues and eigenvectors of $Y_{k_{x}}$. Then the maximally localized states along $y$, known as hybrid Wannier functions, are constructed according to

$$
\left|h_{k_{x} \alpha}\right\rangle=\sum_{n} \xi_{k_{x} \alpha, n}\left|\psi_{k_{x} n}\right\rangle
$$

As we shall see, the spatial locations of their Wannier centers $\bar{y}_{k_{x} \alpha}$ cluster in groups of $J$ per unit cell along $y$, corresponding roughly to the locations along $y$ of the true 2D WFs assigned to a unit cell. Thus, we relabel $\bar{y}_{k_{x} \alpha} \rightarrow \bar{y}_{k_{x} \ell_{y} n}$ and $\left|h_{k_{x} \alpha}\right\rangle \rightarrow\left|h_{k_{x} \ell_{y} n}\right\rangle$, where $\ell_{y}$ is a layer index specifying the unit cell along $y$ and $n=\{1, \ldots, J\}$ labels the Wannier bands within a layer.

Then, for each layer that has been identified in this way, we treat the entire layer as a multiband group, and carry out a maximal localization procedure in the extended direction. To do so, we transform to a twisted parallel transport gauge, i.e., one that makes the the Berry connections $\left\langle\tilde{h}_{k_{x} \ell_{y} n}\left|i \partial_{k_{x}}\right| \tilde{h}_{k_{x} \ell_{y} n^{\prime}}\right\rangle$ diagonal and $k_{x^{-}}$ independent, where $\left|\tilde{h}_{k_{x} \ell_{y} n}\right\rangle=e^{-i k_{x} x}\left|h_{k_{x} \ell_{y} n}\right\rangle$. The fully localized WFs are constructed from the Fourier transform in Eq. (50), thus arriving at WFs that are exponentially localized in both directions. The computation of $q_{x y}^{\mathrm{I}}$ from deep interior WFs, and $d_{x}^{\mathrm{T}}$ from skin-region WFs, then proceeds as described in the previous subsection.

We note in passing that another option for computing $d_{x}^{\mathrm{T}}$ is to bypass the second maximal localization step and simply compute it from Berry phases, as was done in Ref. [23]. That is, having constructed the $\left|\tilde{h}_{k_{x} \ell_{y} n}\right\rangle$, we compute the Berry phases

$$
\gamma_{\ell_{y} n}^{(x)}=\int d k_{x}\left\langle\tilde{h}_{k_{x} \ell_{y} n}\left|i \partial_{k_{x}}\right| \tilde{h}_{k_{x} \ell_{y} n}\right\rangle
$$


on a discretized $k_{x}$ mesh using standard methods. In this context the last term in Eq. (26) becomes $(-e / 2 \pi) \sum_{\ell_{y} n}^{\mathrm{T}} \gamma_{\ell_{y} n}^{(x)}$, where the sums are restricted to the cells associated with the top-edge tiles. However, we find in practice that $P_{x}^{\mathrm{T}}$ computed in this way converges more slowly with respect to $k$-mesh density than does the method based on the direct summation of WF dipoles, which we have therefore adopted below.

\section{Longitudinal-first nested Wannier construction}

The nested procedure outlined in the previous subsection consists of a sequence of two maximal localization steps, the first along the ribbon's finite direction $y$ and the second along the extended direction $x$. If we reverse the order of those two operations, we again arrive at fully localized WFs, albeit in a different gauge. Since the first localization step is now along the extensive direction of the ribbon, we refer to this as the longitudinal-first nested Wannier construction. We note that a similar construction was used in Refs. [4, 5], although the subsequent steps making use of the construction were different there.

We again start from the Bloch eigenstates $\left|\psi_{k_{x} n}\right\rangle$ of Eq. (46). We first transform all of them to a twisted parallel transport gauge in the extensive direction $x$, and then carry out the Fourier transform

$$
\left|h_{\ell_{x} n}^{\prime}\right\rangle=\frac{a}{2 \pi} \int d k_{x} e^{-i k_{x} \ell_{x} a}\left|\psi_{k_{x} n}\right\rangle .
$$

These new states are maximally localized along $x$, but typically they are extended across the width of the ribbon in the $y$ direction. In a sense, they can still be regarded as a species of hybrid WFs. Those with the same index $n$ but different cell indices $\ell_{x}$ are translational copies of one another along $x$. Finally we localize along $y$ the $N_{y} J$ hybrid Wannier functions in each horizontal cell $\ell_{x}$ by performing the steps in Eqs. (51-53) with $\left|\psi_{k_{x} n}\right\rangle$ therein replaced by $\left|h_{\ell_{x} n}^{\prime}\right\rangle$. This yields a set of fully localized WFs $\left|w_{\ell_{x} \ell_{y} n}\right\rangle$, from which the interior quadrupole $q_{x y}^{\mathrm{I}}$ and edge dipoles $d_{x}^{\mathrm{T}}$ can be evaluated as described below Eq. (50).

\section{Quantum distance between Wannier gauges}

Once specific gauges have been chosen for differently oriented ribbons or different Wannier constructions, it is useful to check whether those gauges are consistent. By "consistent gauges" we mean that the sets $\left\{\left|w_{\text {int }, n}\right\rangle\right\}$ and $\left\{\left|\widetilde{w}_{\text {int }, n}\right\rangle\right\}$ of $J$ WFs in one interior cell span the same Hilbert space in both cases. If so, the two sets of WFs are related by a $J \times J$ unitary transformation

$$
\left|\widetilde{w}_{\mathrm{int}, n}\right\rangle=\sum_{m=1}^{J} U_{m n}\left|w_{\mathrm{int}, m}\right\rangle
$$

that only mixes WFs within the same interior cell. On the other hand, Eqs. (36-38) show that $\mathcal{Q}_{x y}^{\mathrm{I}}, \mathcal{P}_{x}^{\mathrm{T}}$, and $\mathcal{P}_{y}^{\mathrm{R}}$ only change under gauge transformations that mix WFs belonging to different cells $\left(\mathbf{R}^{\prime} \neq \mathbf{0}\right)$. This means that we are allowed to evaluated the corner charge as the sum of those three quantities provided that they are evaluated using gauges for the two ribbons that are consistent in the above sense.

The degree of "gauge inconsistency" can be quantified by measuring the "quantum distance" between the two sets of interior WFs. Here the square of the quantum distance $D$ is defined as [29]

$$
\begin{aligned}
D^{2} & =J-\operatorname{Tr}\left[\mathcal{P}_{\text {int }} \widetilde{\mathcal{P}}_{\text {int }}\right] \\
& =J-\sum_{m, n=1}^{J}\left|\left\langle w_{\text {int }, m} \mid \widetilde{w}_{\text {int }, n}\right\rangle\right|^{2}
\end{aligned}
$$

where $\mathcal{P}_{\text {int }}$ and $\widetilde{\mathcal{P}}_{\text {int }}$ are the projection operators onto each set. A vanishing $D$ indicates that the two sets are related by a unitary transformation. Allowing for numerical error, we take the gauges to be consistent whenever $D<10^{-5}$.

\section{RESULTS}

We study three tight-binding models of increasing complexity. All models are centrosymmetric and spinless, and we consider them at half filling. The first is a twoband model (one occupied band), and the other two are four-band models (two occupied bands). In the first two models the symmetry is sufficiently low that the corner charge is not quantized, while the third model has a highsymmetry phase where the corner charge is quantized to either zero or $e / 2$, depending on the choice of parameters. For ribbons and finite flakes, edges are always constructed by simply truncating the bulk, i.e., the hoppings to vacant sites are removed while other hoppings and site energies are unchanged.

In this section, we restrict ourselves to a comparison of the transverse-first nested Wannier construction as applied to both ribbons, as in Ref. [23], and the projection construction. In Sec. VI we will return to the fourband model of Sec. V B and consider the gauge-consistent nested Wannier construction, i.e., $y$-first (or $x$-first) for both ribbons, and show that this also yields a consistent gauge and a correct prediction of the corner charge.

\section{A. Two-band model}

The first model we consider was introduced in Ref. [23], and is illustrated in Fig. 4(a). The rectangular unit cell (gray square) has an aspect ratio of $b / a=0.8$, and contains two atoms along its diagonal, with reduced coordinates $\left(-\frac{1}{6},-\frac{1}{6}\right)$ and $\left(+\frac{1}{6},+\frac{1}{6}\right)$ relative to the center of 
(a)

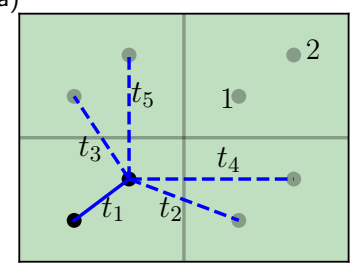

(b)

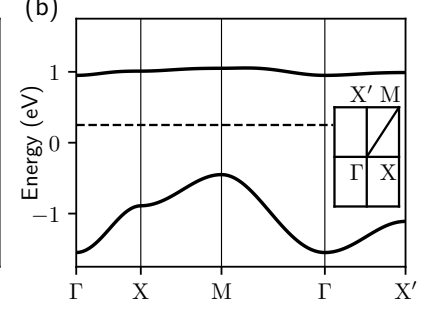

(a)

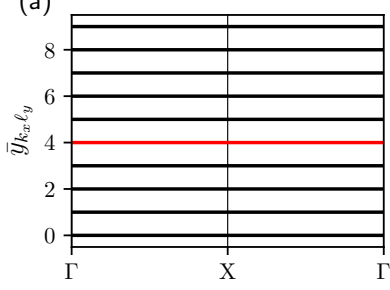

(b)

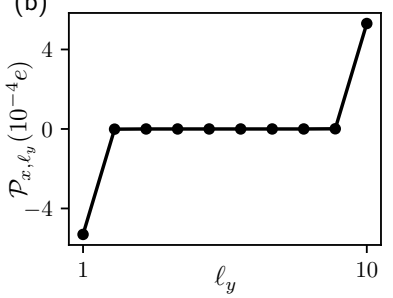

FIG. 4. (a) Visualization of the two-band model. Atoms in the home cell are shown as black dots, the intracell hopping $t_{1}$ as a solid line, and intercell hoppings $t_{2}$ to $t_{5}$ as dashed lines. (b) Bulk band structure for the parameters given in the main text. The Fermi level (dashed line) has been placed at midgap. The inset shows the $2 \mathrm{D}$ Brillouin zone and the high-symmetry points $\Gamma(0,0), \mathrm{X}\left(\frac{1}{2}, 0\right), \mathrm{X}^{\prime}\left(0, \frac{1}{2}\right)$, and $\mathrm{M}$ $\left(\frac{1}{2}, \frac{1}{2}\right)$.

inversion in the middle of the cell. Since we treat the model as spinless and at half filling, we assign a positive charge of $+e / 2$ to each atom to neutralize the unit cell.

Our choice of bulk tile corresponds to the contents of the unit cell in Fig. 4(a), with the reference position $\mathfrak{t}_{n}$ chosen at the origin, which is also the location of the WF center. As a result, the ionic part of the interior quadrupole $q_{x y}^{\mathrm{I}}$ of Eqs. (23-24) is immediately given as $q_{x y}^{\text {ion }}=(e / 36) a b$. The electronic contribution $q_{x y}^{\text {el }}$ in Eq. (25) is determined by the shape asymmetry of the WF charge distribution around its center, and remains to be calculated, as do the dipoles of the edge tiles. From these, $\mathcal{Q}_{x y}^{\mathrm{I}}, \mathcal{P}_{x}^{\mathrm{T}}$, and $\mathcal{P}_{y}^{\mathrm{R}}$ are trivially obtained from Eqs. (12-14).

To evaluate these quantities we construct two ribbons spanning ten unit cells along the $x$ and $y$ directions respectively. We begin by applying the transverse-first nested Wannier construction of Sec. IV B 2 to both ribbons. That is, the maximal localization procedure is first carried out along the finite direction of the ribbon to generate hybrid WFs, and then along the extended direction. The result is illustrated in Fig. 5 for the $y$-finite ribbon. Panel (a) shows the Wannier centers $\bar{y}_{k_{x} \ell_{y}}=\left\langle h_{k_{x} \ell_{y}}|y| h_{k_{x} \ell_{y}}\right\rangle$ obtained in the first step. In the second step, an optimally-smooth gauge along $x$ is enforced within each hybrid Wannier band, resulting in fully localized WFs. Panel (b) shows the layer-resolved dipole moment density along $x$; as expected, it vanishes in the interior region and assumes equal and opposite values at the two edges.

The values of $\mathcal{P}_{x}^{\mathrm{T}}, \mathcal{P}_{y}^{\mathrm{R}}$, and $\mathcal{Q}_{x y}^{\mathrm{I}}$ calculated from those WFs are indicated in the left column of Table I. We find that $\mathcal{Q}_{x y}^{\mathrm{I}}$ has the same value in the two ribbons, suggesting that their gauges are consistent. Decomposing $\mathcal{Q}_{x y}^{\mathrm{I}}$ into ionic and electronic parts, we find $\mathcal{Q}_{x y}^{\text {ion }}=q_{x y}^{\text {ion }} / a b=$ $e / 36 \approx 0.027778 e$ and $\mathcal{Q}_{x y}^{\mathrm{el}}=q_{x y}^{\mathrm{el}} / a b=-0.027808 e$. We also find that the corner charge predicted from Eq. (14) is in excellent agreement with that obtained from a di-
FIG. 5. (a) Wannier bands (i.e, hybrid Wannier centers) $\bar{y}_{k_{x} \ell_{y}}$ (in units of $b$ ) for a ribbon of the two-band model with a width of ten unit cells along $y$. The red Wannier band deep inside the ribbon is selected to construct the fully localized interior Wannier function that is used to evaluate $\mathcal{Q}_{x y}^{\mathrm{I}}$. (b) Layerresolved dipole density $\mathcal{P}_{x, \ell_{y}}=d_{x, \ell_{y}} / a$, computed from the dipole moments of the fully localized Wannier functions in each layer.

rect calculation on a $10 \times 10$ flake using Eq. (44), again suggesting that the gauges are consistent (as well as validating our formalism). The last row of Table I lists the value of the bare corner charge, obtained by simply adding up the charges inside the $5 \times 5$ tiles forming the top-right quadrant of the flake, according to Eq. (45); as expected, the bare corner charge differs significantly from the macroscopic corner charge listed in the two rows above it.

To confirm that the gauges are consistent between the two ribbons, we calculate the quantum distance $D$ according to Eq. (57), and find that it is zero to numerical accuracy. Since there is a single WF per cell, gauge consistency means that the WFs deep inside the two ribbons are the same up to an overall phase factor. The site amplitudes of one such interior WF are listed in Table II.

Recall that the transverse-first nested Wannier construction is not guaranteed to yield consistent gauges for two differently oriented ribbons of a generic model. The

TABLE I. The values of $\mathcal{P}_{x}^{\mathrm{T}}, \mathcal{P}_{y}^{\mathrm{R}}$, and $\mathcal{Q}_{x y}^{\mathrm{I}}$, calculated for ribbons of the two-band model using the transverse-first hybrid Wannier and projection methods. In the bottom half of the table, the corner charge $Q_{\mathrm{c}}$ predicted from Eq. (14) is compared with the value obtained from a direct calculation on a finite flake using Eq. (44), and with the "bare" corner charge obtained from Eq. (45).

\begin{tabular}{lcc}
\hline \hline & $\begin{array}{c}\text { Hybrid Wannier } \\
\left(10^{-3} e\right)\end{array}$ & $\begin{array}{c}\text { Projection } \\
\left(10^{-3} e\right)\end{array}$ \\
\hline $\mathcal{P}_{x}^{\mathrm{T}}$ & -0.531575 & -0.531574 \\
$\mathcal{P}_{y}^{\mathrm{R}}$ & -1.164427 & -1.164427 \\
$\mathcal{Q}_{x y}^{\mathrm{I}}$ & -0.030068 & -0.030068 \\
\hline$Q_{\mathrm{c}}$ (predicted) & -1.726070 & -1.726069 \\
$Q_{\mathrm{c}}$ (direct) & -1.726068 & -1.726068 \\
$Q_{\mathrm{c}}^{\text {bare }}$ & -0.071873 & -0.071873 \\
\hline \hline
\end{tabular}


TABLE II. The bulk-like Wannier function $\left|w_{\text {int }}\right\rangle$ in the home unit cell of the two-band model. $\left|\phi_{\mathbf{R}_{j}}\right\rangle$ is the basis orbital at site $\mathbf{R}+\boldsymbol{\tau}_{j}$, given in reduced coordinates. The 12 largest coefficients are listed; only half of them are shown, as the other half can be obtained by an inversion operation.

\begin{tabular}{crcc}
\hline \hline $\mathbf{R}+\boldsymbol{\tau}_{j}$ & $\left\langle\phi_{\mathbf{R} j} \mid w_{\text {int }}\right\rangle$ & $\mathbf{R}+\boldsymbol{\tau}_{j}$ & $\left\langle\phi_{\mathbf{R}_{j}\left|w_{\text {int }}\right\rangle}\right.$ \\
\hline$\left(-\frac{1}{6},-\frac{1}{6}\right)$ & 0.70565 & $\left(-\frac{5}{6}, \frac{1}{6}\right)$ & 0.02662 \\
$\left(-\frac{7}{6},-\frac{1}{6}\right)$ & -0.02634 & $\left(-\frac{1}{6}, \frac{5}{6}\right)$ & 0.01777 \\
$\left(-\frac{1}{6},-\frac{7}{6}\right)$ & -0.01752 & $\left(\frac{7}{6}, \frac{7}{6}\right)$ & 0.00328 \\
\hline \hline
\end{tabular}

reason why it does so for this particular model is the following. In addition to spatial inversion, the model has time-reversal symmetry, and in the presence of both symmetries the $k$-space Berry curvature of each band vanishes identically. Since the curvature is the curl of the connection, it follows that both the $x$ and $y$ components of the Berry connection can be chosen to be constant. Moreover, these constant values are a measure of the electric polarization, which vanishes here. Thus, in this case of a single occupied band with inversion and time-reversal symmetry, there is a unique "natural" gauge with vanishing Berry connection. This same gauge is arrived at regardless of whether maximal localization is applied first in $x$ and then in $y$, first in $y$ then in $x$, jointly as in conventional 2D maximal localization, or using the projection technique discussed next. ${ }^{4}$

We now repeat the calculations using the projection method of Sec. IV B 1 to fix the gauge. We choose as the trial function the eigenstate of an isolated tile, without any inter-cell hoppings. The trial function in the home unit cell is then $\frac{1}{\sqrt{2}}\left|\phi_{1}\right\rangle+\frac{1}{\sqrt{2}}\left|\phi_{2}\right\rangle$, where $\left|\phi_{1}\right\rangle$ and $\left|\phi_{2}\right\rangle$ are the basis orbitals located at $\left(-\frac{1}{6},-\frac{1}{6}\right)$ and $\left(\frac{1}{6}, \frac{1}{6}\right)$, respectively. After confirming that the resulting gauges for the two ribbons are consistent $(D=0$ to numerical accuracy), we have recalculated $\mathcal{P}_{x}^{\mathrm{T}}, \mathcal{P}_{y}^{\mathrm{R}}$, and $\mathcal{Q}_{x y}^{\mathrm{I}}$, obtaining the values in the right column of Table I. They are identical to the ones in the left column, confirming that the transverse-first hybrid Wannier and projection methods yield consistent gauges for this model. To further verify this, we measure the quantum distance between the interior WFs obtained with the two methods, again obtaining $D=0$.

We conclude by commenting on the results obtained in Ref. [23] for the same model. In that work, $\mathcal{P}_{x}^{\mathrm{T}}$ and $\mathcal{P}_{y}^{\mathrm{R}}$ were calculated for $y$ - and $x$-finite ribbons using the transverse-first nested Wannier construction, and $\mathcal{P}_{x}^{\mathrm{T}}+\mathcal{P}_{y}^{\mathrm{R}}$ was found to be in good agreement with a direct calculation of $Q_{\mathrm{c}}$ for a flake. Our analysis reveals an oversight in that work, also pointed out in Ref. [15],

\footnotetext{
4 The order of the two Wannierization steps becomes irrelevant when the projected position operators $P x P$ and $P y P$ commute, which was shown in Appendix C of Ref. [21] to occur if and only if the Berry curvature vanishes identically.
}

(a)

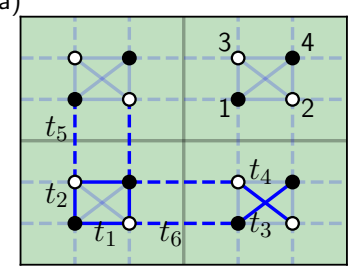

(b)

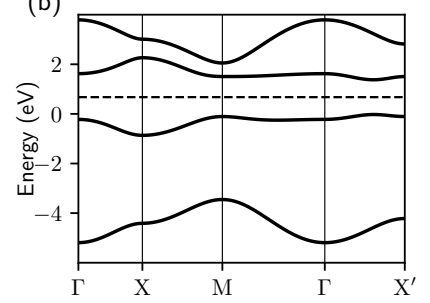

FIG. 6. (a) Visualization of the four-band model. Atoms are labeled from 1 to 4 as shown at upper right. The intracell hoppings $t_{1}, t_{2}, t_{3}$ and $t_{4}$ are shown as solid lines, while the intercell hoppings $t_{5}$ and $t_{6}$ are shown as dashed lines. Sites denoted by open and filled circles have on-site energies $\pm \delta$ respectively. (b) Band structure of the model. The Fermi energy (dashed line) has been placed at midgap.

namely the omission of the $\mathcal{Q}_{x y}^{\mathrm{I}}$ term in Eq. (14). For the choice of parameters in Ref. [23], $\left|\mathcal{Q}_{x y}^{\mathrm{I}}\right|$ is much smaller than both $\left|\mathcal{P}_{x}^{\mathrm{T}}\right|$ and $\left|\mathcal{P}_{y}^{\mathrm{R}}\right|$, helping to explain why that omission was not revealed by the numerical tests carried out there. Reference [23] also neglected to discuss the gauge-consistency issue that arises in more general cases, although as discussed above, it is not a problem for single-occupied-band models with time-reversal symmetry. It does become an issue for multiband cases, as we shall see in our next example.

\section{B. Four-band model}

Our second test case is the model depicted in Fig. 6(a). The unit cell is rectangular with $b / a=0.8$ as before, but it now contains four atoms instead of two, with reduced coordinates $\left(-\frac{1}{6},-\frac{1}{6}\right),\left(\frac{1}{6},-\frac{1}{6}\right),\left(\frac{1}{6}, \frac{1}{6}\right)$ and $\left(-\frac{1}{6}, \frac{1}{6}\right)$ relative to the center of inversion in the middle of the cell. The hopping amplitudes are $t_{1}=-2.0, t_{2}=-1.5$, $t_{3}=-0.8$, and $t_{4}=-0.6 \mathrm{eV}$ (intracell hoppings), and $t_{5}=-0.5$ and $t_{6}=-0.4 \mathrm{eV}$ (intercell hoppings). The sites depicted as open and filled circles have onsite energy $\pm \delta$, where $\delta=0.8$. The band structure is shown in Fig. 6(b); at half filling the two lowest bands are occupied, and we assign a charge of $+e / 2$ to each atom to render the cell neutral. The bulk tile again corresponds to the unit cell, and the reference positions of Eqs. (2122) are again $\mathfrak{t}_{1}=\mathfrak{t}_{2}=\mathbf{0}$; now $q_{x y}^{\text {ion }}=0$ and only $q_{x y}^{\text {el }}$ will contribute to $q_{x y}^{\mathrm{I}}$.

As in our previous example, the model has both spatial inversion and time-reversal symmetry. However, since we now have two occupied bands, the transverse-first nested Wannier construction is no longer expected to produce consistent gauges for the two ribbons. Its application to a 20-cell-thick $y$-finite ribbon is illustrated in Fig. 7. Panel (a) shows the Wannier bands obtained in the first step, with the two bands in each vertical cell being closer to one another than to their neighbors in adjacent cells. 
(a)

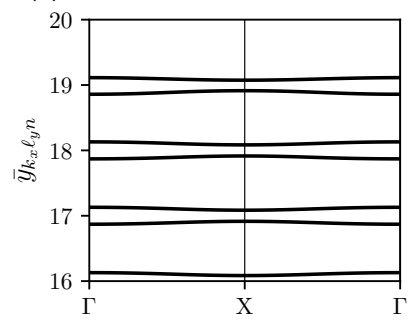

(b)

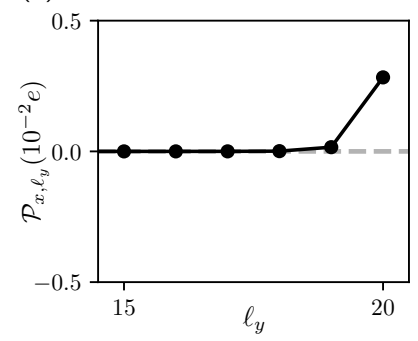

FIG. 7. (a) Wannier bands $\bar{y}_{k_{x} \ell_{y} n}$ (in units of $b$ ) for a $y$-finite ribbon of the four-band model with a width of 20 layers, and two bands per layer. (b) Dipole moment density of the layers near the top edge of the ribbon.

In the second step, the maximal localization procedure is applied along $x$, treating the two hybrid Wannier functions within a cell as a composite group, resulting in two fully localized WFs per 2D cell. When applied to the $x$-finite ribbon, the transverse-first procedure results in a similar pair of WFs, but now obtained by localizing first along $x$ and then along $y$.

The center column of Table III lists the calculated values of $\mathcal{P}_{x}^{\mathrm{T}}$ (for the $y$-finite ribbon), $\mathcal{P}_{y}^{\mathrm{R}}$ (for the $x$-finite ribbon), and $\mathcal{Q}_{x y}^{\mathrm{I}}$ (for both). Even though $\mathcal{Q}_{x y}^{\mathrm{I}}$ has identical values in both ribbons, the predicted corner charge $Q_{\text {c }}$ differs by about $0.14 \%$ from that obtained via a direct calculation on a $20 \times 20$ flake, indicating some degree of gauge inconsistency. The gauges of the two ribbons are indeed slightly different, as can be seen by inspecting the second and third columns of Table IV, where we list the site amplitudes of one of the two interior WFs per cell (the other is related to it by spatial inversion) in each ribbon. To check that this difference cannot be accounted for by a $2 \times 2$ intracell gauge transformation described by Eq. (56), we calculate the quantum distance of Eq. (57) to be $D=0.0138$. This nonzero value confirms that the interior gauges produced by this naive hybrid Wannier

TABLE III. The values of $\mathcal{P}_{x}^{\mathrm{T}}, \mathcal{P}_{y}^{\mathrm{R}}, \mathcal{Q}_{x y}^{\mathrm{I}}$, calculated for ribbons of the four-band model using the transverse-first hybrid Wannier and projection methods. In the center column, the identical value of $\mathcal{Q}_{x y}^{\mathrm{I}}$ found for both ribbons is reported. In the last two rows, the corner charge $Q_{\mathrm{c}}$ predicted from Eq. (14) is compared with the value obtained from a direct calculation on a finite flake.

\begin{tabular}{lcr}
\hline \hline & $\begin{array}{c}\text { Hybrid Wannier } \\
\left(10^{-2} e\right)\end{array}$ & $\begin{array}{c}\text { Projection } \\
\left(10^{-2} e\right)\end{array}$ \\
\hline $\mathcal{P}_{x}^{\mathrm{T}}$ & 0.300250 & 0.254669 \\
$\mathcal{P}_{y}^{\mathrm{R}}$ & 0.476420 & 0.446029 \\
$\mathcal{Q}_{x y}^{\mathrm{I}}$ & -3.756016 & -3.684265 \\
\hline$Q_{\mathrm{c}}$ (predicted) & -2.979346 & -2.983567 \\
$Q_{\mathrm{c}}$ (direct) & -2.983567 & -2.983567 \\
\hline \hline
\end{tabular}

TABLE IV. One of the two bulk-like Wannier functions in the home unit cell of the four-band model, constructed in three different ways. $\left|w_{\text {int }, 1}^{(y)}\right\rangle$ and $\left|w_{\text {int, } 1}^{(x)}\right\rangle$ are obtained by applying the transverse-first nested Wannier construction to $y$ and $x$-finite ribbons, respectively, while $\left|w_{\mathrm{int}, 1}^{(\mathrm{p})}\right\rangle$ is obtained by applying the projection method to both ribbons starting from the trial function $\left|g_{1}\right\rangle$ described in the main text. $\left|\phi_{\mathbf{R} j}\right\rangle$ is the basis orbital at site $\mathbf{R}+\boldsymbol{\tau}_{j}$, given in reduced coordinates.

\begin{tabular}{ccccc}
\hline \hline $\mathbf{R}+\boldsymbol{\tau}_{j}$ & $\left\langle\phi_{\mathbf{R} j} \mid w_{\text {int, } 1}^{(y)}\right\rangle$ & $\left\langle\phi_{\mathbf{R} j} \mid w_{\text {int, } 1}^{(x)}\right\rangle$ & $\left\langle\phi_{\mathbf{R} j} \mid w_{\text {int, } 1}^{(\mathrm{p})}\right\rangle$ & $\left\langle\phi_{\mathbf{R} j} \mid g_{1}\right\rangle$ \\
\hline$\left(-\frac{1}{6},-\frac{1}{6}\right)$ & -0.86557 & -0.86563 & -0.86481 & -0.87128 \\
$\left(-\frac{1}{6}, \frac{1}{6}\right)$ & -0.42664 & -0.42656 & -0.42851 & -0.45897 \\
$\left(\frac{1}{6},-\frac{1}{6}\right)$ & -0.17659 & -0.17654 & -0.17732 & -0.15379 \\
$\left(-\frac{1}{6},-\frac{5}{6}\right)$ & -0.11485 & -0.11527 & -0.10720 & 0 \\
$\left(-\frac{5}{6},-\frac{1}{6}\right)$ & -0.07271 & -0.07294 & -0.07163 & 0 \\
$\left(\frac{1}{6}, \frac{1}{6}\right)$ & 0.07108 & 0.07108 & 0.07185 & 0.08101 \\
$\left(\frac{1}{6}, \frac{5}{6}\right)$ & 0.06598 & 0.06548 & 0.06286 & 0 \\
$\left(\frac{5}{6}, \frac{1}{6}\right)$ & 0.04861 & 0.04798 & 0.04626 & 0 \\
\hline \hline
\end{tabular}

approach is inconsistent between the two ribbons.

To arrive at a common gauge for the two ribbons we use the projection method, choosing as trial functions $\left|g_{1}\right\rangle=\frac{1}{\sqrt{2}}\left|\psi_{1}\right\rangle+\frac{1}{\sqrt{2}}\left|\psi_{2}\right\rangle$ and $\left|g_{2}\right\rangle=\frac{1}{\sqrt{2}}\left|\psi_{1}\right\rangle-\frac{1}{\sqrt{2}}\left|\psi_{2}\right\rangle$, where $\left|\psi_{1}\right\rangle$ and $\left|\psi_{2}\right\rangle$ are the two lowest-energy eigenstates of an isolated tile, i.e., with intercell hoppings set to zero. These two eigenstates are of even and odd parity respectively, so that $\left|g_{1}\right\rangle$ and $\left|g_{2}\right\rangle$ are each off-centered with respect to the origin, and map into one another under inversion.

Applying the projection method to ribbon models cut from the bulk as described in Sec. IV B 1, we find as expected that the pair of WFs taken from the deep interior of the $x$-finite ribbon match those extracted from the $y$ finite ribbon within numerical precision. We denote as $\left|w_{\text {int }, 1}^{(\mathrm{p})}\right\rangle$ and $\left|w_{\text {int,2 }}^{(\mathrm{p})}\right\rangle$ the WFs projected from $\left|g_{1}\right\rangle$ and $\left|g_{2}\right\rangle$ respectively. Like the trial functions, these lie off-center and map into one another under inversion. In the last two columns of Table IV we list the site amplitudes of $\left|w_{\text {int, } 1}^{(\mathrm{p})}\right\rangle$ and $\left|g_{1}\right\rangle$. It is evident that the projected WFs are similar, but not identical, to the ones obtained by the transverse-first hybrid Wannier approach; we find a quantum distances of $D=0.03844$ and 0.03857 respectively from the projected pair to the pairs generated via the transverse-first nested Wannierization of $x$-finite and $y$-finite ribbons respectively.

Having verified that the projection method leads to two ribbons described by the same bulk gauge, we proceeded to calculate $\mathcal{P}_{x}^{\mathrm{T}}$ for the $y$-finite ribbon and $\mathcal{P}_{y}^{\mathrm{R}}$ for the $x$ finite ribbon; their values are listed in the right column of Table III, followed by the common value of $\mathcal{Q}_{x y}^{\mathrm{I}}$ in both ribbons. In contrast to the center column, the sum of the three now matches perfectly the value of $Q_{\mathrm{c}}$ in the finite flake.

This example confirms our expectation that the corner charge can reliably be predicted from ribbon calcu- 
(a)

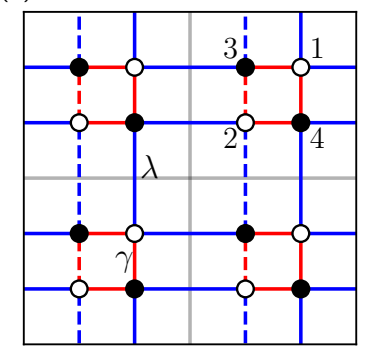

(b)

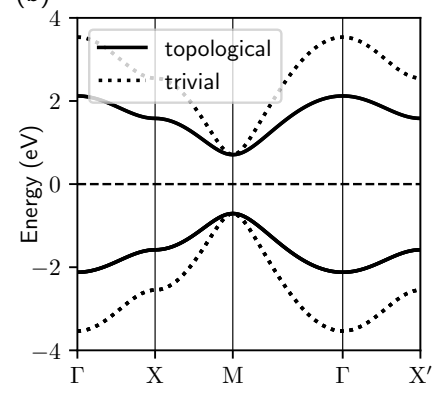

FIG. 8. (a) Visualization of the Benalcazar-Bernevig-Hughes model. Atoms are labeled from 1 to 4 inside the unit cell, as shown at upper right. Intracell hoppings of amplitude $\pm \gamma$ are shown as solid or dashed red (short) lines, and intercell hoppings of amplitude $\pm \lambda$ as solid or dashed blue (long) lines. In the calculations reported in the main text, sites denoted by open and filled circles have small on-site energies $\pm \delta$ respectively. (b) Band structure of the model for $\lambda=1.0, \delta=0$, and two different values of $\gamma$. The solid bands were calculated at $\gamma=1.5$ (trivial phase, $Q_{\mathrm{c}}=0$ ), and the dashed ones at $\gamma=0.5$ (topological phase, $Q_{\mathrm{c}}=e / 2$ ). In both cases, the bands are doubly degenerate. The Fermi energy (dashed line) has been placed at midgap.

lations alone, provided that consistent gauges are used for both ribbons, even in the case of multiple occupied bands. It also illustrates the fact that this gauge consistency is achieved only via the projection method, ${ }^{5}$ while the transverse-first hybrid Wannier approach fails in this case.

\section{Benalcazar-Bernevig-Hughes (BBH) model}

Our final test case is a model introduced by Benalcazar, Bernevig, and Hughes as an example of a topological phase with quantized corner charges $[4,5]$. The BBH model is pictured in Fig. 8(a). It has four sites per cell as in our previous example, but now placed on a square lattice. We again choose the atoms to have reduced coordinates $\left(-\frac{1}{6},-\frac{1}{6}\right),\left(\frac{1}{6},-\frac{1}{6}\right),\left(\frac{1}{6}, \frac{1}{6}\right)$ and $\left(-\frac{1}{6}, \frac{1}{6}\right)$ relative to the origin at the center of a small square. ${ }^{6}$ Figure $8(\mathrm{a})$ shows four unit cells (gray squares) centered in the same way, but as we shall see later, our choice of bulk tile may or may not coincide with this unit cell. Each site also carries an ionic charge of $+e / 2$, so that the system is neutral at half filling.

\footnotetext{
${ }^{5}$ A gauge-fixing method was recently proposed in Ref. [15] based on parallel transport as intercell hoppings are varied. Although this was applied only to a single-occupied-band case, we expect that this method, while more complicated than ours, would also lead to bulk-like WFs in a multi-band case.

6 The location of the sites was not specified in Refs. [4 and 5]. Our choice of $1 / 6$ is arbitrary.
}

When viewed along $x$ or $y$, the model consists of parallel chains with dimerized bonds. The hopping amplitudes along $x$ alternate between $\gamma$ (intracell) and $\lambda$ (intercell). The same bond alternation occurs along $y$, except that the hopping amplitudes change sign from one chain to the next, as though $\pi$ fluxes have been threaded through the plaquettes. Following BBH, we also include an optional parameter $\delta$ which, if present, assigns an on-site energy $\pm \delta$ to the sites depicted as open and filled circles respectively in Fig. 8(a).

The model always has inversion and time-reversal symmetry, and in the absence of $\delta$ it also has $M_{x}$ and $M_{y}$ mirror and $C_{4}$ rotational symmetries. (Strictly speaking, the spatial symmetry operators only return the system to itself after a sign-flip gauge change, but this does not affect the symmetry arguments.) The BBH model was introduced largely for the purpose of investigating the consequences of symmetry for the bare model $(\delta=0)$. The BBH and subsequent papers have shown that the presence of $M_{x}$ and $M_{y}$ symmetries, or $C_{4}$ symmetry, constrains the corner charge of a rectangular flake to be a multiple of $e / 4$ quite generally, or of $e / 2$ in some cases $[4,5,8,12-14,30]$, stimulating interest in the theory of higher-order topological phases [6]. We can understand this in the context of our Eq. (14) by noting that $\mathcal{P}_{x}^{\mathrm{T}}=-\mathcal{P}_{y}^{\mathrm{R}}$ and $\mathcal{Q}_{x y}=0$ in a $C_{4}$-respecting gauge, leaving only the $Q^{\mathrm{TR}}$ contribution of Eq. (28). For a general rectangular-lattice system, this must be either zero or a multiple of $e / 4(\bmod e)$, depending on whether any fractional ionic charges were left over in the corner tile after the bulk and edge tiling. (In the context of the $\mathrm{BBH}$ model, $C_{4}$ symmetry implies $Q_{\mathrm{c}}=0$ or $e / 2$.)

Here, instead, we are more interested in the case that spatial symmetries other than inversion are not present, so that the corner charge is not quantized. Returning to the BBH model, at $\delta=0$ the model has two gapped phases, a trivial phase with $Q_{\mathrm{c}}=0$ for $|\gamma / \lambda|>1$ and a topological phase with $Q_{\mathrm{c}}= \pm e / 2$ for $|\gamma / \lambda|<1$. The bulk energy gap closes at the $\mathrm{M}$ point in the $\mathrm{BZ}$ at the critical $|\gamma / \lambda|=1$. In what follows a small $\delta$ is applied to break the mirror and $C_{4}$ symmetries. Note that we continue to refer to the resulting systems as being in the "trivial" or "topological" phase, even though such a classification is no longer strictly well defined.

\section{Trivial and topological phases}

In our calculations we set $\lambda=1.0$, and choose $\gamma=1.5$ and $\gamma=0.5$ to put the system in the trivial and topological phases, respectively. The resulting energy dispersions, plotted in Fig. 8(b), consist of two doublydegenerate bands separated by finite gaps. To fix the sign of the corner charge in the topological phase, $\mathrm{BBH}$ weakly broke the quantizing symmetries $M_{x}, M_{y}$ and $C_{4}$ while preserving inversion symmetry by adding a nonzero $\delta$ term to the Hamiltonian $[4,5]$. When $\delta$ is small, $Q_{\mathrm{c}}$ deviates slightly from the quantized value. The results 
(a)

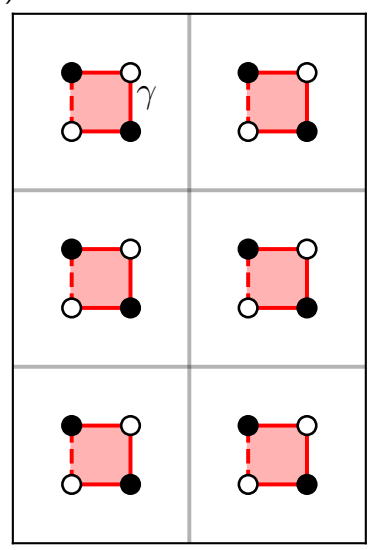

(b)

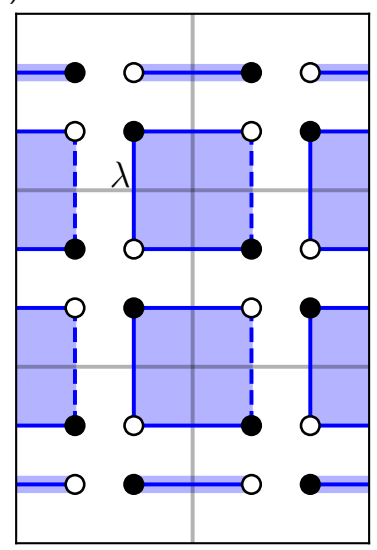

FIG. 9. Visualization of the isolated tiles whose low-energy eigenstates serve as trial functions for constructing Wannier functions in ribbons of the Benalcazar-Bernevig-Hughes model. The figures represent $y$-finite ribbons three unit cells high, while those used in the actual calculations are 40 unit cells high. (a) Tiles used for the trivial phase (red squares). (b) Tiles used for the topological phase. The blue squares are interior tiles. At the edges and corners, there are "left-over" dimers and isolated atoms, respectively.

reported below are obtained using $\delta=0.001$ for both phases. Since the model has two occupied bands, we know from our previous example that the transverse-first nested Wannier construction cannot be trusted to produce consistent gauges for the two ribbons, so we focus here on the projection approach from the outset.

In view of the qualitative difference between the trivial and topological phases, we adopt a different choice of bulk tile for each case. For the trivial phase we choose the bulk tile to correspond to the unit cell centered on the small red square in Fig. 9(a), with reference locations $\mathfrak{t}_{1}=\mathfrak{t}_{2}=\mathbf{0}$. The ionic interior quadrupole in Eq. (24) is thus $q_{x y}^{\text {ion }}=0$, with $q_{x y}^{\text {el }}$ in Eq. (25) left to be determined by the anisotropy of the Wannier charge distribution. Since we assume the sample has been cut as shown in Fig. 8(a), there will be no left-over charges in the top-right corner tile, i.e., $Q^{\mathrm{TR}}=0$.

By contrast, for the topological phase, the WFs are chosen to be associated with a large blue square in Fig. 9(b), e.g., the one centered at $(1 / 2,1 / 2)$. A choice of tile involving these WFs together with ions centered around $(0,0)$ would generate a bulk dipole, which our approach requires us to avoid. Instead, we choose the bulk tile as the unit cell centered on the large blue square in Fig. 9(b), with reference positions $\mathfrak{t}_{1}=\mathfrak{t}_{2}=(1 / 2,1 / 2)$ at the center of this square. ${ }^{7}$ Again the symmetry is such that $q_{x y}^{\text {ion }}=0$ in Eq. (24), and $q_{x y}^{\text {el }}$ in Eq. (25) is left to be

\footnotetext{
${ }^{7}$ Note that here, if we had taken a model with ionic charge $2 e$
}

TABLE V. Bulk-like Wannier functions in the home unit cell of the BBH model in the trivial phase. The Wannier functions are constructed using the projection method, choosing as trial orbitals $\left|g_{1}\right\rangle$ and $\left|g_{2}\right\rangle$ the lowest-energy eigenstates of the isolated red-square tile in Fig. $9(\mathrm{a}) .\left|\phi_{\mathbf{R} j}\right\rangle$ is the basis orbital located at site $\mathbf{R}+\boldsymbol{\tau}_{j}$, given in reduced coordinates.

\begin{tabular}{ccccc}
\hline \hline $\mathbf{R}+\boldsymbol{\tau}_{j}$ & $\left\langle\phi_{\mathbf{R} j} \mid w_{\mathrm{int}, 1}^{(\mathrm{p})}\right\rangle$ & $\left\langle\phi_{\mathbf{R} j} \mid g_{1}\right\rangle$ & $\left\langle\phi_{\mathbf{R} j} \mid w_{\mathrm{int}, 2}^{(\mathrm{p})}\right\rangle$ & $\left\langle\phi_{\mathbf{R} j} \mid g_{2}\right\rangle$ \\
\hline$\left(\frac{1}{6}, \frac{1}{6}\right)$ & 0.67899 & 0.70694 & 0 & 0 \\
$\left(-\frac{1}{6},-\frac{1}{6}\right)$ & 0 & 0 & 0.67899 & 0.70694 \\
$\left(-\frac{1}{6}, \frac{1}{6}\right)$ & -0.48037 & -0.50012 & 0.48037 & 0.50012 \\
$\left(\frac{1}{6},-\frac{1}{6}\right)$ & -0.48037 & -0.50012 & -0.48037 & -0.50012 \\
$\left(\frac{5}{6}, \quad \frac{1}{6}\right)$ & -0.12012 & 0 & -0.03317 & 0 \\
$\left(\frac{1}{6}, \frac{5}{6}\right)$ & -0.12012 & 0 & 0.03317 & 0 \\
$\left(-\frac{5}{6},-\frac{1}{6}\right)$ & 0.03317 & 0 & -0.12012 & 0 \\
$\left(-\frac{1}{6},-\frac{5}{6}\right)$ & 0.03317 & 0 & 0.12012 & 0 \\
$\left(\frac{5}{6},-\frac{1}{6}\right)$ & 0.10844 & 0 & 0.06157 & 0 \\
$\left(-\frac{1}{6}, \quad \frac{5}{6}\right)$ & -0.10844 & 0 & 0.06157 & 0 \\
$\left(-\frac{5}{6}, \frac{1}{6}\right)$ & 0.06157 & 0 & -0.10844 & 0 \\
$\left(\frac{1}{6},-\frac{5}{6}\right)$ & 0.06157 & 0 & 0.10844 & 0 \\
$\left(\frac{1}{6}, \frac{7}{6}\right)$ & -0.05019 & 0 & -0.00333 & 0 \\
$\left(\frac{7}{6}, \quad \frac{1}{6}\right)$ & -0.05019 & 0 & 0.00333 & 0 \\
$\left(-\frac{1}{6},-\frac{7}{6}\right)$ & 0.00333 & 0 & -0.05019 & 0 \\
$\left(-\frac{7}{6},-\frac{1}{6}\right)$ & -0.00333 & 0 & -0.05019 & 0 \\
\hline \hline
\end{tabular}

determined. Note that there are now two left-over ionic charges that need to be assigned to each top tile as shown in Fig. 9(b), and similarly for the right edge tiles. Each corner tile acquires one ionic charge of $+e / 2$, so $Q^{\mathrm{TR}}$ of the corner tile will be $-e / 2$ if there is an occupied WF in that tile and $+e / 2$ otherwise. From a minimal knowledge of the model, we can anticipate that a WF will be present in the top-right tile if and only if $\delta<0$.

To obtain gauge-consistent values for $\mathcal{P}_{x}^{\mathrm{T}}, \mathcal{P}_{y}^{\mathrm{R}}$, and $\mathcal{Q}_{x y}^{\mathrm{I}}$ via projection, we begin by considering a $y$-finite ribbon 40 unit cells high, with simple periodic boundary conditions along $x$. The trial functions are chosen as the low-energy eigenstates of the isolated tiles obtained by removing the weaker of the two hoppings. For the trivial phase, we take as trial functions the two lowest-energy eigenstates of the isolated small red square in Fig. 9, replicated 40 times to cover the entire ribbon. For the topological phase the WF centers shift to the large blue squares $[7,9,10]$, so we take their isolated eigenstates as our projection functions, replicated 39 times. We also include two edge tiles, one at the top and one at the bottom of the ribbon, each consisting of a single dimer with its single low-energy eigenstate. Taken together, these states comprise our trial functions for the ribbon in the

at the origin from the outset, we would have needed to use the split-basis approach discussed below Eq. (17), yielding the same pattern of fractional charges. This is not the case for the trivial phase. 
TABLE VI. Same as Table V, but for the topological phase of the BBH model. The trial orbitals $\left|g_{1}\right\rangle$ and $\left|g_{2}\right\rangle$ are now chosen as the lowest-energy eigenstates of the isolated bluesquare tile in Fig. 9(b).

\begin{tabular}{ccccc}
\hline \hline $\mathbf{R}+\boldsymbol{\tau}_{j}$ & $\left\langle\phi_{\mathbf{R} j} \mid w_{\mathrm{int}, 1}^{(\mathrm{p})}\right\rangle$ & $\left\langle\phi_{\mathbf{R} j} \mid g_{1}\right\rangle$ & $\left\langle\phi_{\mathbf{R} j} \mid w_{\mathrm{int}, 2}^{(\mathrm{p})}\right\rangle$ & $\left\langle\phi_{\mathbf{R} j} \mid g_{2}\right\rangle$ \\
\hline$\left(\frac{1}{6}, \quad \frac{1}{6}\right)$ & 0.69081 & 0.70686 & 0 & 0 \\
$\left(\frac{5}{6}, \quad \frac{5}{6}\right)$ & 0 & 0 & 0.69081 & 0.70686 \\
$\left(\frac{5}{6}, \quad \frac{1}{6}\right)$ & -0.48885 & -0.50018 & 0.48885 & 0.50018 \\
$\left(\frac{1}{6}, \quad \frac{5}{6}\right)$ & -0.48885 & -0.50018 & -0.48885 & -0.50018 \\
$\left(-\frac{1}{6}, \quad \frac{1}{6}\right)$ & -0.09152 & 0 & -0.02753 & 0 \\
$\left(\frac{1}{6},-\frac{1}{6}\right)$ & -0.09152 & 0 & 0.02753 & 0 \\
$\left(\frac{7}{6}, \frac{5}{6}\right)$ & 0.02753 & 0 & -0.09152 & 0 \\
$\left(\frac{5}{6}, \quad \frac{7}{6}\right)$ & 0.02753 & 0 & 0.09152 & 0 \\
$\left(\frac{5}{6},-\frac{1}{6}\right)$ & -0.08424 & 0 & 0.04535 & 0 \\
$\left(-\frac{1}{6}, \quad \frac{5}{6}\right)$ & 0.08424 & 0 & 0.04535 & 0 \\
$\left(\frac{1}{6}, \quad \frac{7}{6}\right)$ & 0.04535 & 0 & 0.08424 & 0 \\
$\left(\frac{7}{6}, \quad \frac{1}{6}\right)$ & 0.04535 & 0 & -0.08424 & 0 \\
$\left(-\frac{5}{6}, \quad \frac{1}{6}\right)$ & -0.04049 & 0 & 0.00160 & 0 \\
$\left(\frac{1}{6},-\frac{5}{6}\right)$ & -0.04049 & 0 & -0.00160 & 0 \\
$\left(\frac{11}{6}, \quad \frac{5}{6}\right)$ & -0.00160 & 0 & -0.04049 & 0 \\
$\left(\frac{5}{6}, \quad \frac{11}{6}\right)$ & 0.00160 & 0 & -0.04049 & 0 \\
\hline \hline
\end{tabular}

topological phase. We do the same for $x$-finite ribbons, and we confirm that within each phase, the deep interior WFs are identical for $x$ - and $y$-finite ribbons. The site amplitudes of the resulting WFs are given in Table V for the trivial phase, and in Table VI for the topological phase, together with the trial functions for comparison.

From the consistent sets of WFs obtained for the two ribbons, we calculate edge polarizations and interior quadrupoles in the usual manner. To accommodate the left-over dimer WFs in the outermost layers in the topological phase, the edge polarizations are evaluated from edge tiles containing an odd number of WFs, while in the trivial phase that number is even. The values of $\mathcal{P}_{x}^{\mathrm{T}}$, $\mathcal{P}_{y}^{\mathrm{R}}$, and $\mathcal{Q}_{x y}^{\mathrm{I}}$ are listed in Table VII. These are all very small, of order $10^{-5} e$ and $10^{-4} e$ in the trivial and topological phases respectively, as a consequence of the small $\delta$. The fourth contribution $Q^{\mathrm{TR}}$ vanishes in the trivial phase and is $e / 2$ in the topological phase. Summing all four contributions, we find excellent agreement with the directly calculated macroscopic corner charge in both phases. Thus, in both cases, the small deviation from the quantized $Q_{\mathrm{c}}$ value caused by the staggered on-site potential is precisely reproduced by the ribbon calculations.

\section{Corner charge pumping cycle}

In this section, we carry out calculations of the interior quadrupole and edge polarizations, and compare the predicted corner charge with the directly calculated one,
TABLE VII. Individual contributions and total predicted macroscopic corner charge $Q_{\mathrm{c}}$ in Eq. (14), compared with a direct calculation, for the trivial and topological phases of the BBH model as depicted in Fig. 8(b). In both cases, the symmetries that quantize the corner charge are weakly broken by a staggered on-site potential (see main text). The values of $\mathcal{P}_{x}^{\mathrm{T}}, \mathcal{P}_{y}^{\mathrm{R}}$, and $\mathcal{Q}_{x y}^{\mathrm{I}}$ are obtained from ribbon calculations, while $Q^{\mathrm{TR}}$ is inferred mod $e$ from the tiling procedure. The last line reports the bare corner charge computed by summing over the top-right quadrant.

\begin{tabular}{lcc}
\hline \hline & Trivial & Topological \\
\hline $\mathcal{P}_{x}^{\mathrm{T}}$ & $0.854 \times 10^{-5}$ & $-44.077 \times 10^{-5}$ \\
$\mathcal{P}_{y}^{\mathrm{R}}$ & $0.854 \times 10^{-5}$ & $-44.077 \times 10^{-5}$ \\
$\mathcal{Q}_{x y}^{\mathrm{I}}$ & $4.517 \times 10^{-5}$ & $18.412 \times 10^{-5}$ \\
$Q^{\mathrm{TR}}$ & 0 & 0.5 \\
\hline$Q_{\mathrm{c}}($ predicted $)$ & $6.225 \times 10^{-5}$ & $0.5-69.743 \times 10^{-5}$ \\
$Q_{\mathrm{c}}($ direct $)$ & $6.225 \times 10^{-5}$ & $0.5-69.743 \times 10^{-5}$ \\
$Q_{\mathrm{c}}^{\text {bare }}$ & $1.602 \times 10^{-5}$ & $0.5-84.817 \times 10^{-5}$ \\
\hline \hline
\end{tabular}

for the same adiabatic cycle

$$
(\delta, \lambda, \gamma)=\left\{\begin{array}{cl}
(\cos (t), \sin (t), 0) & 0<t \leqslant \pi \\
(\cos (t), 0,|\sin (t)|) & \pi<t \leqslant 2 \pi
\end{array}\right.
$$

considered previously by BBH $[4,5]$. This cycle is somewhat artificial, in that one or the other of the hoppings $\gamma$ or $\lambda$ is always zero. However, to make contact with previous literature, we apply our method to the same system here.

At $t=0$ the system starts in a state in which the sites are completely decoupled, with only black sites in Fig. 9 occupied as a result of the positive $\delta$. In the interval $0<t<\pi$, a set of positive $\lambda$ hoppings are first turned on and then turned off on the edges of the large blue squares in Fig. 9. In this interval, the system takes the form of a molecular crystal with "molecules" centered on the large blue squares. At $t=\pi / 2$ where $\delta$ vanishes, the symmetry suffices to define the topological index, and the system is in the nontrivial phase. Once $t$ passes $\pi / 2$ the sign of $\delta$ is reversed, so that at $t=\pi$ we again reach a state of completely decoupled sites, but now with only the open-circle sites occupied. The second half of the loop is similar, except that now the $\gamma$ hoppings are progressively turned on and off, so that the system is molecular once more, but centered on the small red squares. The topology is again defined at $t=3 \pi / 2$, now being trivial, and the system returns to its starting point at $t=2 \pi$.

We use two different sets of trial functions for the Wannier projection during the first and second halves of the cycle. For $t \in[0, \pi]$ we adopt the trial functions of the topological state, while for $t \in[\pi, 2 \pi]$ we choose those of the trivial state, as described in the previous subsection and detailed in Tables VI and V respectively. We thus have a gauge discontinuity at $t=\pi$ and again at $t=2 \pi$. For a mesh of $t$ values, we compute $\mathcal{Q}_{x y}^{\mathrm{I}}, \mathcal{P}_{x}^{\mathrm{T}}$, and $\mathcal{P}_{y}^{\mathrm{R}}$, and compare the prediction of Eq. (14) with the directly 


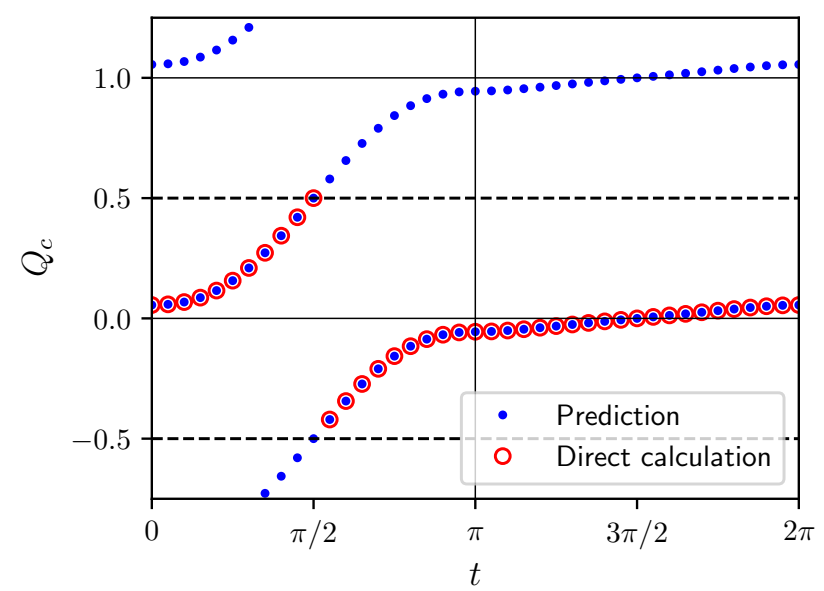

FIG. 10. Evolution of the corner charge $Q_{\mathrm{c}}$ (in units of $e$ ) during the adiabatic pumping cycle described by Eq. (58). The ribbon calculations only predict $Q_{\mathrm{c}}$ modulo $e$, so three branches are plotted vs. $t$ as the blue dots. The evolution of the actual corner charge of a finite flake is indicated by the red circles.

computed macroscopic corner charge of a large but finite flake. The results are presented in Fig. 10. Since the corner charge is predicted only $\bmod e$, we plot several branches corresponding to the periodicity of $e$ along the vertical axis as blue dots, and the directly calculated corner charges are the red circles.

We confirm that $Q_{\mathrm{c}}=e / 2$ and zero $(\bmod e)$ at $\pi / 2$ and $3 \pi / 2$ respectively, where the topology is sharply defined. However, we find that most of the pumping of the corner charge occurs in the first half of the cycle. That is, $Q_{\mathrm{c}}$ grows from $e / 18$ to $17 e / 18$ in this interval, for an increase of $8 e / 9$, while the growth in the second half of the cycle is only by the remaining amount $e / 9$.

Note that the gauge discontinuities at $t=\pi$ and $2 \pi$ introduce no discontinuities in the predicted value of $Q_{\mathrm{c}}$. However, there are discontinuities in the individual values of $\mathcal{Q}_{x y}^{\mathrm{I}}, \mathcal{P}_{x}^{\mathrm{T}}$, and $\mathcal{P}_{y}^{\mathrm{R}}$. In the first half of the cycle, $\mathcal{Q}_{x y}^{\mathrm{I}}$ comes from the larger blue-square tile and changes from $2 e / 9$ to $-2 e / 9$, while in the second half $\mathcal{Q}_{x y}^{\mathrm{I}}$ comes from the twice-smaller red-square tile and grows from $-e / 18$ to $e / 18$. In the first half-cycle, $\mathcal{P}_{x}^{\mathrm{T}}=\mathcal{P}_{y}^{\mathrm{R}}$ each increase from $-e / 3$ to $e / 3$, while in the second half $\mathcal{P}_{x}^{\mathrm{T}}$ and $\mathcal{P}_{y}^{\mathrm{R}}$ are identically zero. Finally, our tiling is such that $Q^{\mathrm{TR}}=$ $e / 2(\bmod e)$ in the first half-cycle, and zero $(\bmod e)$ in the second half. Adding the various contributions according to Eq. (14), we find that the total $Q_{\mathrm{c}}$ evolves as described in the previous paragraph.

Without a knowledge of the population of WFs in the corner tile, we can only make predictions "mod $e$ " as done above. In particular, we cannot predict precisely when the corner charge will make the discontinuous jump needed to allow it to return to its initial state at the end of the pumping cycle. However, by inspecting the
Hamiltonian, we can anticipate that a WF will be present in the top-right tile in the interval $\pi / 2<t<\pi$, when the open circle at top right in Fig. 9(b) has negative energy, but not otherwise. Making use of this additional information about $Q^{\mathrm{TR}}$, we expect the discontinuity in the macroscopic corner charge to occur at $t=\pi / 2$. We then correctly predict not only the value $\bmod e$, but also the correct branch choice, of $Q_{\mathrm{c}}$ over the entire cycle.

A comparison of our Fig. 10 with Fig. 37 of Ref. [5], which also compares predictions from ribbons with a computed corner charge, shows important differences. In their case, all the change in the corner charge occurs in the first half-cycle, when it evolves from 0 to $e$, and there is no change in the second half-cycle. While the computed corner charges agree with the predictions in their theory, as they do in ours, it is important to keep in mind that the two approaches differ in crucial ways. (i) In Ref. [5], BBH do not compute the macroscopic corner charge defined by Eq. (43); instead, they compute the total charge of the upper-right quadrant, that is, the bare corner charge of Eq. (45). In fact, since they did not specify the positions of the orbitals, the macroscopic corner charge is ill-determined in their case. For the trivial and topological cases discussed in Sec. V C 1, we obtain the values of $Q_{c}^{\text {bare }}$ presented in the last row of Table VII, which are clearly very different from the macroscopic corner charges. ${ }^{8}$ (ii) Their edge polarizations $p^{\text {edge }}$ are not defined in the same way as ours. For the specified cycle, their $p^{\text {edge }}$ is defined in such a way that $d p^{\text {edge }} / d t$ corresponds to the flow of current into a quadrant, while our $d \mathcal{P} / d t$ corresponds to the polarization current associated with the changing dipole moments of the edge tiles in the skin region. (iii) In our theory, in order to correctly predict the macroscopic corner charge, we also insist that bulk quadrupole and surface dipole contributions are computed in a common Wannier gauge. As a result of these differences, each theory obtains internally consistent results, although we argue that ours is more physical in that it predicts a macroscopically observable corner charge.

\section{GAUGE-CONSISTENT NESTED WANNIER CONSTRUCTION}

In the previous section, we demonstrated that a naive application of the hybrid Wannier approach, in which the transverse-first nested Wannier construction is applied to ribbons of both orientations, is not gauge-consistent,

\footnotetext{
8 If all sites are located precisely at the origin in the middle of the unit cell, the bare and macroscopic corner charges become equal. This follows because $W(x, y)$ in Eq. (44) is identical for all electronic and ionic charges in the cell, and the total charge of the cell vanishes both for deep interior cells and for skin cells far from the corners. Thus, Eq. (44) is equivalent to integrating the charge density over a quadrant.
} 
whereas an alternative projection construction does result in a consistent gauge. Here, we demonstrate a second successful method for generating a consistent gauge, this time without the need for providing trial functions. We do this using the nested Wannier constructions described in Secs. IV B 2 and IV B 3, but now insuring that the two localization steps are executed in the same order for both the $x$-finite and $y$-finite ribbons. In other words, one should apply the transverse construction of Sec. IV B 2 to one ribbon, and the longitudinal construction of Sec. IV B 3 to the other.

Let us apply this procedure to the four-band model of Fig. 6, for which we obtained inconsistent gauges in Sec. V B by applying the transverse construction to both ribbons. We choose to localize first along $y$ and then along $x$. Thus we apply the same transverse construction as before to the $y$-finite ribbon, and apply the longitudinal construction to the $x$-finite ribbon. We find that deep inside the two ribbons the resulting WFs are identical: within numerical accuracy, their site amplitudes are the same and the quantum distance between them vanishes. We then repeat the entire procedure but localizing first along $x$ and then along $y$, and again we arrive at the same interior gauge for both ribbons (but different from the previous one).

Table VIII shows the individual contributions and total predicted corner charge in the two nested Wannier gauges. The predicted corner charges are the same in both, and they agree perfectly with the actual corner charge of a finite flake. Note that while the edge polarizations are different between those two gauges, the interior quadrupoles are identical. The reason is that $\mathcal{Q}_{x y}^{\mathrm{I}}$ is a symmetric tensor, and hence it remains unchanged upon reversing the order of the $x$ and $y$ localization steps.

We have also tested this gauge-consistent nested Wannier approach for the $\mathrm{BBH}$ model $[4,5]$, and we again find that the corner charge is correctly predicted. The implementation is straightforward following the example of the four-band-model discussed above.

Before concluding the discussion of this method, we note that it is possible to bypass the second step of the longitudinal-first construction. Briefly, again working in the $y$-first context, we carry out only the first step of the $y$-first construction for the $x$-finite ribbon. We identify the total charge $\rho_{\ell_{y}}(\mathbf{r})$ of the ions and WFs associated with any one of the single-cell-high layers $\ell_{y}$, and compute its $y$-dipole density $d^{(y)}(x)=\int y \rho_{\ell_{y}}(x, y) d y$. This quantity is independent of $\ell_{y}$, and letting $\bar{d}^{(y)}(x)$ be its window average in the $x$ direction, we note that $\bar{d}^{(y)}(x)$ vanishes except near the edges of the ribbon, and its integral over the right skin region gives $\mathcal{Q}_{x y}^{\mathrm{I}}+\mathcal{P}_{y}^{\mathrm{R}}$. Adding this to the $\mathcal{P}_{x}^{\mathrm{T}}$ obtained from the transverse-first nested Wannier construction for the $y$-finite ribbon then gives the correct corner charge as before. Nevertheless, we recommend applying the two-step nested procedure to both ribbons, as this increases the reliability of the method by allowing a cross-check on the equivalence of the two sets of WFs.
TABLE VIII. Individual contributions and total predicted macroscopic corner charge $Q_{\mathrm{c}}$ in Eq. (14), compared with a direct calculation, for the four-band model of Fig. 6 . The ribbon calculations were performed using a gauge-consistent nested Wannier construction where we first Wannierize along $y$ and then along $x$ (middle column), or vice-versa (right-column).

\begin{tabular}{lcc}
\hline \hline & $\begin{array}{c}\text { Wannierize } y \text { then } x \\
\left(10^{-2} e\right)\end{array}$ & $\begin{array}{c}\text { Wannierize } x \text { then } y \\
\left(10^{-2} e\right)\end{array}$ \\
\hline $\mathcal{P}_{x}^{\mathrm{T}}$ & 0.300250 & 0.296029 \\
$\mathcal{P}_{y}^{\mathrm{R}}$ & 0.472198 & 0.476420 \\
$\mathcal{Q}_{x y}^{\mathrm{I}}$ & -3.756016 & -3.756016 \\
\hline$Q_{\mathrm{c}}$ (predicted) & -2.983567 & -2.983567 \\
$Q_{\mathrm{c}}$ (direct) & -2.983567 & -2.983567 \\
\hline \hline
\end{tabular}

\section{DISCUSSION}

Several generalizations of our work remain to be developed. Our current formulation is trivially extended to the case of broken time-reversal symmetry, and the presence of spinor electrons entails no special difficulty. The case of nonrectangular crystals and corner angles other than $90^{\circ}$ can be treated following the methods of Ref. [15]. By contrast, generalizations to topological systems, such as 2D Chern insulators or $Z_{2}$-odd quantum spin Hall insulators, do not look straightforward. In these cases, metallic edge states are topologically protected, interfering with any natural definition of edge polarization. Finally, while we have focused here on the case of low-symmetry systems such that the corner charge is not quantized, further exploration of the connections to the theory of higherorder topological insulators in higher-symmetry systems is desirable.

Generalizations to higher dimensions are easily anticipated. The line of intersection of two surface facets of a 3D crystal, generally known as a "hinge," carries a linear charge density that can be computed via an elementary extension of the present methods, either by Wannierizing in all three dimensions, or by Wannierizing in 2D at each $k_{\|}$(wavevector along the hinge) and averaging over $k_{\|}$. The prediction of the corner charge in $3 \mathrm{D}$, while perhaps more difficult in practice, should follow the same principles outlined here. That is, one would need to compute the octupoles of interior bulk tiles far from any surfaces, the quadrupoles of surface tiles far from any hinges, and the dipoles of hinge tiles. While these will not be individually gauge-invariant, their sum will be, allowing for a prediction of the corner charge $\bmod e$. So, for example, a calculation of three rectangular rod geometries, one each extending along $\hat{\mathbf{x}}, \hat{\mathbf{y}}$, and $\hat{\mathbf{z}}$, should provide all the needed information.

Throughout this work we have assumed the presence of bulk inversion symmetry so that the bulk cell can be chosen to be free of an electric dipole moment. However, other symmetries can also force a nonpolar point group. In $2 \mathrm{D}$ these would be the $C_{6}, C_{4}$, and $C_{3}$ rotations $\left(C_{2}\right.$ is 
equivalent to inversion in 2D). All of these $C_{n}$ symmetries force $q_{x y}^{\mathrm{I}}$ to vanish, and result in quantized corner charges for a crystallite in the shape of a regular $n$-gon. However, there could be cases of inequivalent edges meeting, as for example a $90^{\circ}$ corner of a material with bulk $C_{3}$ symmetry. In such cases the adjoining edges are inequivalent and could result in a generic corner charge. More opportunities arise for nonpolar but noncentrosymmetric point groups in $3 \mathrm{D}$. It should be straightforward to generalize our theory to such cases.

We end this section with a discussion of connections to the theory or orbital magnetization, which we already briefly invoked to argue that surface polarization is not a physical observable. We argued that if it were, its time derivative ought to correspond to a physical flow of current at the edge of the 2D sample. However, for a timereversal broken system with a nonzero orbital magnetization, a steady current circulates around the edges of the sample, which is inconsistent with a uniquely defined edge polarization. By contrast, it is clear that the edge current is a physical observable; it can be evaluated as an expectation value of a Hermitian operator in the usual way, and is fully gauge-invariant.

There is a strong formal similarity between the theory presented here and that developed by Thonhauser et al. [31] and Ceresoli et al. [32] to derive the moderntheory expression for orbital magnetization using the Wannier representation. In fact, that work made use of an identical decomposition of the Wannier functions of a large but finite flake into those associated with interior and skin regions, and identified two contributions to the orbital magnetization. One, denoted as the "local circulation," was identified with the internal circulation of charge in a deep-interior WF. The second, labeled "itinerant circulation," arises from edge currents defined as the expectation value of the current operator traced over WFs in the skin region. The current of this type on the right-hand edge, labeled as $I_{y}$ in Ref. [31] and denoted as $I_{y}^{\mathrm{R}}$ henceforth, is just the time derivative of the edge polarization $\mathcal{P}_{y}^{\mathrm{R}}$ defined here. Indeed the expression for $I_{y}^{\mathrm{R}}$ in Eq. (9) of Ref. [31] takes the form of a sum of contributions from hoppings that cross the boundary between the interior and skin regions, just as our expression in Eq. (38) for the change in $\mathcal{P}_{y}^{\mathrm{R}}$ under a gauge change depends on lattice vectors $\mathbf{R}^{\prime}$ crossing that same boundary.

This is no accident. Since we are in the ground state, the unitary time-evolution operator $e^{-i H t / \hbar}$ does not change the occupied subspace, but it does modify the gauge by multiplying each energy eigenstate by a phase factor $e^{-i E t / \hbar}$. An infinitesimal time step $\delta t$ corresponds to an infinitesimal unitary transformation in which the deep interior WFs change by $\delta|\mathbf{0} m\rangle=\sum_{\mathbf{R}^{\prime} n} \epsilon_{\mathbf{R}^{\prime}, n m}\left|\mathbf{R}^{\prime} n\right\rangle$, using a notation consistent with Eq. (30), with

$$
\epsilon_{\mathbf{R}^{\prime}, n m}=-i \frac{\delta t}{\hbar}\left\langle\mathbf{R}^{\prime} n|H| \mathbf{0} m\right\rangle .
$$

Substituting into Eq. (38) and using Eq. (34), the upward-flowing current $I_{y}^{\mathrm{R}}=\delta \mathcal{P}_{y}^{\mathrm{R}} / \delta t$ on the right edge of the sample is

$$
I_{y}^{\mathrm{R}}=\frac{2 e}{a b} \frac{1}{\hbar} \sum_{R_{x}^{\prime}>0} \sum_{n m} R_{x}^{\prime}\left\langle\mathbf{0} m|y| \mathbf{R}^{\prime} n\right\rangle\left\langle\mathbf{R}^{\prime} n|H| \mathbf{0} m\right\rangle .
$$

In other words, time evolution within the occupied subspace generates a gauge evolution, and the changing gauge drives a displacement of WF centers in the skin region that corresponds precisely to the itinerant edge current $I_{y}^{\mathrm{R}}$. Equation (60) reproduces the expressions derived in Refs. [31] and [32] for the single-band and multiband cases respectively. The (counterclockwise) itinerant-circulation contribution to the orbital magnetization is given by the average of $I_{y}^{\mathrm{R}}$ on the right edge and $-I_{x}^{\mathrm{T}}$ on the top edge, while instead the difference between $I_{y}^{\mathrm{R}}$ and $-I_{x}^{\mathrm{T}}$ (that is, $I_{y}^{\mathrm{R}}+I_{x}^{\mathrm{T}}$ ) corresponds to a skin contribution to the time rate of change of the topright corner charge. The latter is in fact independent of time, so this must be exactly canceled by a contribution from the time dependence of the interior-tile Wannier quadrupole, which is more closely related to the local circulation in the orbital magnetization theory.

These relationships indicate a deep formal connection between the theory of orbital magnetization and that of edge polarizations and corner charges presented here.

\section{SUMMARY}

In summary, we have considered the case of a $2 \mathrm{D}$ centrosymmetric insulator in which the corner charges are not quantized by additional symmetries. Decomposing the large but finite flake into bulk, skin, and corner regions, and introducing a tiling in this context, we have shown that the corner charge can be written as a sum of a quadrupole contribution associated with the bulk tiles, and two dipole contributions associated with the two edges that meet at the corner. Having introduced a Wannier representation to attach electron charges to these tiles, we demonstrated that the bulk quadrupole and two edge dipole contributions are not individually gauge-invariant, although their sum is. As a consequence, we argue that it is crucially important to adopt a common gauge for the computation of all of these quantities in the two ribbon geometries.

To verify the correctness of our approach, we have tested it via calculations on three different tight-binding models. We have demonstrated two different methods for arriving at a consistent gauge for ribbons of both orientations, one based on projection from trial functions and another based on a consistently applied nested Wannier construction. We emphasize that the macroscopically observable corner charge has to be computed by an appropriate coarse-graining procedure, and not simply by counting charges in a quadrant of the sample. Having taken all these constraints into account, we have demonstrated that the corner charge can indeed be computed modulo $e$, to numerical accuracy, from calculations on 
two ribbon geometries alone. We are hopeful that our work paves the way toward the emergence of a deeper and more general understanding of the intimate connections between bulk and surface properties of crystalline materials.

\section{ACKNOWLEDGMENTS}

Work by S.R. and D.V. was supported by NSF Grant DMR-1954856. Work by I.S. was supported by Grant No. FIS2016-77188-P from the Spanish Ministerio de Economía y Competitividad.
[1] R. D. King-Smith and David Vanderbilt, "Theory of polarization of crystalline solids," Phys. Rev. B 47, 16511654 (1993).

[2] Raffaele Resta, "Macroscopic polarization in crystalline dielectrics: the geometric phase approach," Rev. Mod. Phys. 66, 899-915 (1994).

[3] D. Vanderbilt, Berry Phases in Electronic Structure Theory (Cambridge University Press, 2018).

[4] W. A. Benalcazar, B. A. Bernevig, and T. L. Hughes, "Quantized electric multipole insulators," Science 357, 61-66 (2017).

[5] W. A. Benalcazar, B. A. Bernevig, and T. L. Hughes, "Electric multipole moments, topological multipole moment pumping, and chiral hinge states in crystalline insulators," Phys. Rev. B 96, 245115 (2017).

[6] Siddharth A Parameswaran and Yuan Wan, "Topological insulators turn a corner," Physics 10, 132 (2017).

[7] Zhida Song, Zhong Fang, and Chen Fang, " $(d-2)$ dimensional edge states of rotation symmetry protected topological states," Phys. Rev. Lett. 119, 246402 (2017).

[8] Guido van Miert and Carmine Ortix, "Higher-order topological insulators protected by inversion and rotoinversion symmetries," Phys. Rev. B 98, 081110 (2018).

[9] Motohiko Ezawa, "Minimal models for wannier-type higher-order topological insulators and phosphorene," Phys. Rev. B 98, 045125 (2018).

[10] Eslam Khalaf, Wladimir A. Benalcazar, Taylor L. Hughes, and Raquel Queiroz, "Boundary-obstructed topological phases," (2019), arXiv:1908.00011 [condmat.mes-hall].

[11] Tianhe Li, Penghao Zhu, Wladimir A. Benalcazar, and Taylor L. Hughes, "Fractional disclination charge in twodimensional $C_{n}$-symmetric topological crystalline insulators," Phys. Rev. B 101, 115115 (2020).

[12] Frank Schindler, Marta Brzezińska, Wladimir A. Benalcazar, Mikel Iraola, Adrien Bouhon, Stepan S. Tsirkin, Maia G. Vergniory, and Titus Neupert, "Fractional corner charges in spin-orbit coupled crystals," Phys. Rev. Research 1, 033074 (2019).

[13] Haruki Watanabe and Seishiro Ono, "Corner charge and bulk multipole moment in periodic systems," Phys. Rev. B 102, 165120 (2020).

[14] Sander Kooi, Guido van Miert, and Carmine Ortix, "The bulk-corner correspondence of time-reversal symmetric insulators," npj Quantum Materials 6 (2021), 10.1038/s41535-020-00300-7.

[15] Luka Trifunovic, "Bulk-and-edge to corner correspondence," Phys. Rev. Research 2, 043012 (2020).
[16] Haruki Watanabe and Hoi Chun Po, "Fractional corner charge of sodium chloride," (2020), arXiv:2009.04845 [cond-mat.mtrl-sci].

[17] Byungmin Kang, Ken Shiozaki, and Gil Young Cho, "Many-body order parameters for multipoles in solids," Phys. Rev. B 100, 245134 (2019).

[18] William A. Wheeler, Lucas K. Wagner, and Taylor L. Hughes, "Many-body electric multipole operators in extended systems," Phys. Rev. B 100, 245135 (2019).

[19] R. Resta, "Quantum-mechanical position operator in extended systems," Phys. Rev. Lett. 80, 1800-1803 (1998).

[20] Seishiro Ono, Luka Trifunovic, and Haruki Watanabe, "Difficulties in operator-based formulation of the bulk quadrupole moment," Phys. Rev. B 100, 245133 (2019).

[21] N. Marzari and D. Vanderbilt, "Maximally localized generalized Wannier functions for composite energy bands," Phys. Rev. B 56, 12847 (1997).

[22] Nicola Marzari, Arash A. Mostofi, Jonathan R. Yates, Ivo Souza, and David Vanderbilt, "Maximally localized Wannier functions: Theory and applications," Rev. Mod. Phys. 84, 1419-1475 (2012).

[23] Yuanjun Zhou, Karin M. Rabe, and David Vanderbilt, "Surface polarization and edge charges," Phys. Rev. B 92, 041102 (2015).

[24] David Vanderbilt and R. D. King-Smith, "Electric polarization as a bulk quantity and its relation to surface charge," Phys. Rev. B 48, 4442-4455 (1993).

[25] Luka Trifunovic, Seishiro Ono, and Haruki Watanabe, "Geometric orbital magnetization in adiabatic processes," Phys. Rev. B 100, 054408 (2019).

[26] Akito Daido, Atsuo Shitade, and Youichi Yanase, "Thermodynamic approach to electric quadrupole moments," Phys. Rev. B 102, 235149 (2020).

[27] The PүтнTB code package is available at http://www.physics.rutgers.edu/pythtb/about.html.

[28] Raffaele Resta, "Towards a bulk theory of flexoelectricity," Phys. Rev. Lett. 105, 127601 (2010).

[29] Jianpeng Liu and David Vanderbilt, "Spin-orbit spillage as a measure of band inversion in insulators," Phys. Rev. B 90, 125133 (2014).

[30] Wladimir A. Benalcazar, Tianhe Li, and Taylor L. Hughes, "Quantization of fractional corner charge in $C_{n}$-symmetric higher-order topological crystalline insulators," Phys. Rev. B 99, 245151 (2019).

[31] T. Thonhauser, Davide Ceresoli, David Vanderbilt, and R. Resta, "Orbital magnetization in periodic insulators," Phys. Rev. Lett 95, 137205 (2005).

[32] D. Ceresoli, T. Thonhauser, D. Vanderbilt, and R. Resta, "Orbital magnetization in crystalline solids: Multi-band insulators, Chern insulators, and metals," Phys. Rev. B 74, 024408 (2006). 